\title{
Reconfigurable Intelligent Surface Assisted Multi-Carrier Wireless Systems for Doubly Selective High-Mobility Ricean Channels
}

\author{
Chao Xu, Senior Member, IEEE, Jiancheng An, Tong Bai, Luping Xiang, Shinya Sugiura, Senior Member, IEEE, \\ Robert G. Maunder, Senior Member, IEEE, Lie-Liang Yang, Fellow, IEEE and Lajos Hanzo* Life Fellow, IEEE
}

\begin{abstract}
Reconfigurable intelligent surfaces (RIS) constitute a revolutionary technique of beneficially reconfiguring the smart radio environment. However, despite the fact that wireless propagation is of time-varying nature, most of the existing RIS contributions focus on time-invariant scenarios for the following reasons. Firstly, it becomes impractical to instantaneously feed back the control signal based on the doubly selective non-line-ofsight (NLoS) fading scenario. Secondly, channel estimation conceived for the high-mobility and high-dimensional RIS-assisted links has to take into account the spatial-domain (SD), timedomain (TD), and frequency-domain (FD) correlations imposed by the angle-of-arrival/departure (AoA/AoD), the Doppler and the orthogonal frequency-division multiplexing (OFDM) operations, respectively, where none of the existing solutions can be directly applied. Thirdly, it is far from trivial to maximize the NLoS channel powers on all subcarriers by a common set of RIS reflecting coefficients. Fourthly, in the face of double selectivity, it becomes inevitable to encounter either inter-symbol interference (ISI) or inter-channel interference (ICI) during the signal detection in the TD or in the FD, respectively. Against this background, firstly, we focus our attention on line-of-sight (LoS) dominated unmanned aerial vehicle (UAV) scenarios. Secondly, we conceive new minimum mean squared error (MMSE) channel estimation methods for doubly selective fading, which perodically transmit pilot symbols embedded into the TD and FD over the SD in order to beneficially exploit the correlations in the three domains. Thirdly, the RIS coefficients are optimized by a low-complexity algorithm based on the LoS representation of the end-to-end system model. Fourthly, tailor-made interference cancallation techniques are devised for improving the signal detection both in the FD and in the TD. Our simulation results are examined in six frequency bands licensed in $5 \mathrm{G}$, which confirms that the employment of RIS is capable of achieving substantial performance improvements.
\end{abstract}

Index Terms-Reconfigurable intelligent surface, channel estimation, high-mobility, double selectivity, inter-symbol interference, inter-channel interference, orthogonal frequency-division multiplexing, space-air-ground, unmanned aerial vehicle.

\section{INTRODUCTION}

The performance of wireless communications is predominantly determined by the signal propagation environment. A

C. Xu, L. Xiang, R. G. Maunder L-L. Yang and L. Hanzo are with the School of Electronics and Computer Science, University of Southampton, Southampton SO17 1BJ, UK (e-mail: \{cx1g08,1x2e19,rm,lly,lh\}@soton.ac.uk). J. An is with the School of Information and Communication Engineering, University of Electronic Science and Technology of China, Chengdu, Sichuan 611731, China (e-mail: jiancheng_an@163.com). S. Sugiura is with the Institute of Industrial Science, University of Tokyo, Meguro-ku, Tokyo 153-8505, Japan (e-mail: sugiura@ieee.org).

L. Hanzo would like to acknowledge the financial support of the Engineering and Physical Sciences Research Council projects EP/P034284/1 and EP/P003990/1 (COALESCE) as well as of the European Research Council's Advanced Fellow Grant QuantCom (Grant No. 789028). signal emitted from a transmitter will arrive at the receiver in form of multiple attenuated and delayed copies, a phenomenon known as the multipath effect [1]-[4]. The majority of wireless communication techniques, including channel coding, modulation, beamforming and diversity schemes [5][7], endeavour to adapt to the multipath effect without having any ability to change the propagation environment. Against this background, the recent developments in meta-materials [8]-[11] inspired the revolutionary notion of smart radio environments, where the signal propagation can be favourably influenced. Specifically, the recently-developed reconfigurable intelligent surfaces (RIS) [12]-[15] consist of a large number of low-cost passive elements, which are managed by a smart micro controller. Each RIS element is configured to apply an adjustable phase rotation to the signal reflection, so that the received signals can be constructively enhanced at the desired user and/or destructively nulled at the non-intended users. To reap the appealling benefits of passive RIS in practical systems, it is of paramount importance to obtain accurate channel state information (CSI) for phase configuration.

The RIS channel estimation methods may be classified into the following four main categories. First of all, the line-of-sight (LoS) based schemes [16]-[19] configure the RIS purely based on the deterministic LoS knowledge of positioning, which leads to sub-optimal solutions. Secondly, the partially active arrangements of [20]-[22] still rely on a small number of RF chains used for sounding the channels for a certain fraction of the RIS elements. Then the full channel knowledge of all RIS elements may be acquired by using compressive sensing and deep learning tools. However, this hybrid philosophy tends to contradict to the motivation of using passive RIS elements, resulting in an increased hardware cost and power consumption. Thirdly, the schemes in [23]-[27] proposed the ON/OFF and discrete Fourier transform (DFT) based RIS patterns for cascaded channel estimation. More explicitly, the ON/OFF based least square (LS) schemes [23], [24] successively estimate the cascaded base station (BS)-RISUser links by activating a single RIS element in a time slot, so that the interference between RIS elements used for channel estimation is avoided. In order to improve this, both the LS and the minimum mean squared error (MMSE) methods of [25]-[27] activate all of the RIS elements based on the classic DFT matrix, so that the direct link and all reflected links become orthogonal, which facilitates their dedicated channel estimation. Fourthly, as the number of RIS elements increases, the pilot overhead of estimating both the direct link and the RIS-reflected links can be further reduced by grouping 
together a set of adjacent RIS elements [23], [28], [29] and upon configuring the RIS based on the statistical knowledge of the CSI [30], by compressive sensing [31], [32], deep learning [33] and matrix factorization [34]-[37]. For multi-user RIS systems, a novel three-phase channel estimation method was proposed in [24], which exploited the commonality of the BSRIS link shared by all users. Furthermore, channel estimation is completely eliminated in [38] by invoking non-coherent modulation, where the RIS is configured to rely on random phases.

At the time of writing, most of the existing RIS treatises focus on time-invariant scenarios, where block-wise quasistatic fading is assumed. However, the wireless communication channels are time-varying in nature due to the mobility of the transmitter, receiver and the surrounding objects. Furthermore, high-mobility scenarios are of crucial importance for future wireless generations providing ubiquitous tetherless connectivity. The first release of the $5 \mathrm{G}$ New Radio (NR) standard supports a highway car velocity of up to $140 \mathrm{~km} / \mathrm{h}$ and high-speed train velocity of $500 \mathrm{~km} / \mathrm{h}$ [39], [40]. The modernization of Air Traffic Management (ATM) is also underway, which aims for improving the existing communication links for aircraft travelling at $1080 \mathrm{~km} / \mathrm{h} \mathrm{[41]-[43].} \mathrm{In} \mathrm{the} \mathrm{much-anticipated} \mathrm{space-air-}$ ground integrated network, both the $5 \mathrm{G}$ networks and the nextgeneration ATM are developing standards for unmanned aerial vehicles (UAVs) popularly known as drones or quadcopters [43]-[45]. The low-altitude rotary-wing UAVs are generally permitted to travel at a speed up to $108 \mathrm{~km} / \mathrm{h}$, while the highaltitude fixed-wing UAVs may exceed the speed of sound. In these scenarios, the associated Doppler frequencies easily reach thousands of Hertz, which is a substantial percentage of the subcarrier spacing (SCS) in the operational orthogonal frequency-division multiplexing (OFDM) standards. This leads to the detrimental effect of doubly-selective fading channels [1]-[7]. More explicitly, on one hand, when the delay-spread of the multipath components exceeds the symbol-level sampling period, inter-symbol interference (ISI) is encountered in frequency-selective scenarios, where the coherence bandwidth becomes lower than the signal bandwidth. We note that the coherence bandwidth defines the range of frequency over which the frequency-domain (FD) channel transfer function is near-constant and this bandwidth is inversely propotional to the mean delay-spread. To mitigate this, OFDM is invoked in order to divide the frequency-selective wideband channel into orthogonal non-dispersive subchannels. On the other hand, the coherence time characterizes the time inverval over which the fading channel's envelope may be deemed near-constant and this duration is inversely propotional to the maximum Doppler frequency. In high-mobility scenarios, the coherence time may become lower than the OFDM symbol duration, which leads to inter-carrier interference (ICI) that destroys OFDM's subcarrier (SC) orthogonality.

Therefore, the deployment of RIS in doubly selective fading channels faces the following major challenges [46]-[50]. First of all, despite the fact that the RIS reconfiguration can be implemented near-instantaneously, where the switching frequency of the positive-intrinsic-negative (PIN) diode may reach $5 \mathrm{MHz}$ [12], it remains a challenge to instantaneously feed back the control signal based on the doubly selective nonline-of-sight (NLoS) fading elements, whose coherence time is lower than the OFDM symbol period. Secondly, channel estimation plays a crucial role for both the RIS configuration and for the signal detection. However, for the high-Doppler and high-dimensional RIS channels, the CSI estimation has to take into account the spatial-domain (SD), time-domain (TD), and FD correlations imposed by the angle-of-arrival/departure (AoA/AoD), the Doppler and the OFDM operations, respectively, where none of the existing solutions can be directly applied. Thirdly, due to the passive nature of RIS, the same phase rotation of each RIS element will be applied to all the channel impulse response (CIR) taps in the TD or equivalently to the channel frequency response (CFR) of all SCs in the FD. As a result, the optimization of the RIS reflection coefficients for maximizing the NLoS channel powers of all SCs remains a non-trivial problem. Finally, in the face of double selectivity, it becomes inevitable to encounter either ISI or ICI during the signal detection in the TD or in the FD, respectively. Moreover, the detrimental ICI increases both with the Doppler frequency and carrier frequency. These open problems call for more advanced interference-mitigation techniques.

Against this background, in this contribution, we propose to mitigate the above-mentioned challenges in the following aspects. First of all, the RIS control link is facilitated by relying on the LoS characteristics. The LoS modelling involves both the positioning information [16]-[19] and the high frequency offset $\Delta f_{\text {LOS }}$ that is jointly determined by the direction and speed of movement. Therefore, the time-varying LoS can be readily predicted and fed back on the control link in advance. Secondly, we propose new MMSE channel estimation methods for both FD and TD signal processing, which are conceived based on a comprehensive analytical model of the superimposed direct BS-User-link and reflected BS-RISUser-link in the face of doubly selective fading. Thirdly, the RIS coefficients are optimized by a low-complexity algorithm based on the LoS characteristics that are known a priori. Finally, upon conceiving MMSE-based channel estimation methods for doubly selective fading, bespoke interference cancallation techniques are devised for improving the signal detection both in FD and in TD.

To elaborate, in Table I we boldly and explicitly contrast our novel contributions to the state-of-the-art RIS-assisted communication systems. In terms of RIS modelling, the three essential features of having passive elements, modelling both direct and reflected links as well as creating favourable LoS propagation are highlighted in Table I, where the existing schemes exhibit specific pros and cons in this regard. In the face of high-mobility, Table I takes into account the adaptivity to AoA/AoD that determines the correlations in the SD, the LoS frequency offset $\Delta f_{\mathrm{LOS}}$ that determines the time-varying nature of the LoS components as well as the normalized maximum Doppler frequency $f_{d}$ that determines the time-varying nature of the NLoS components. The proposed scheme is the first one that includes all of these important high-mobility factors in the analytical model. Furthermore, the proposed solution is also the first one capable of coping with both frequency-selectivity and time-selectivity, as seen in Table I. 


\begin{tabular}{|c|c|c|c|c|c|c|c|c|c|}
\hline & & & RIS modellir & & & ptive to mob & & Doubly & ctivity \\
\hline & $\begin{array}{l}\text { Channel estimation } \\
\text { methods }\end{array}$ & $\begin{array}{l}\text { Passive } \\
\text { RIS? }\end{array}$ & $\begin{array}{l}\text { Both direct and } \\
\text { reflected links? }\end{array}$ & $\begin{array}{l}\text { Considered } \\
\text { LoS? }\end{array}$ & $\begin{array}{l}\text { Adaptive to } \\
\text { AoA/AoD? }\end{array}$ & $\begin{array}{l}\text { Considered } \\
\Delta f_{\mathrm{LOS}} ?\end{array}$ & $\begin{array}{l}\text { Adaptive to } \\
\text { high } f_{d} \text { ? }\end{array}$ & $\begin{array}{l}\text { Frequency- } \\
\text { Selectivity }\end{array}$ & $\begin{array}{l}\text { Time- } \\
\text { Selectivity }\end{array}$ \\
\hline Han et al. [16] & LoS knowledge & $\sqrt{ }$ & $\sqrt{ }$ & $\sqrt{ }$ & $\sqrt{ }$ & & & & \\
\hline Taha et al. [20] & $\begin{array}{l}\text { Compressive } \\
\text { deep learning }\end{array}$ & & & & $\sqrt{ }$ & & & $\sqrt{ }$ & \\
\hline Yang et al. [23] & ON/OFF-based LS & $\sqrt{ }$ & $\sqrt{ }$ & $\sqrt{ }$ & & & & $\sqrt{ }$ & \\
\hline Wang et al. [24] & ON/OFF-based LS & $\sqrt{ }$ & $\sqrt{ }$ & $\sqrt{ }$ & $\sqrt{ }$ & & & & \\
\hline Jensen et al. [25] & DFT-based LS & $\sqrt{ }$ & $\sqrt{ }$ & & & & & & \\
\hline Zheng et al. [26] & DFT-based LS & $\sqrt{ }$ & $\sqrt{ }$ & $\sqrt{ }$ & & & & $\sqrt{ }$ & \\
\hline Nadeemet al. [27] & DFT-based MMSE & $\sqrt{ }$ & $\sqrt{ }$ & $\sqrt{ }$ & $\sqrt{ }$ & & & & \\
\hline You et al. [29] & DFT-based LS & $\sqrt{ }$ & & $\sqrt{ }$ & & & & & \\
\hline Chen et al. [32] & Compressive sensing & $\sqrt{ }$ & & & $\sqrt{ }$ & & & & \\
\hline Ma et al. [33] & Deep learning & $\sqrt{ }$ & & $\sqrt{ }$ & $\sqrt{ }$ & & & & \\
\hline He et al. [34] & Matrix factorization & $\sqrt{ }$ & & & $\sqrt{ }$ & & & & \\
\hline Liu et al. [35] & Matrix factorization & $\sqrt{ }$ & $\sqrt{ }$ & $\sqrt{ }$ & $\sqrt{ }$ & & & & \\
\hline Proposed Scheme & MMSE & $\sqrt{ }$ & $\sqrt{ }$ & $\sqrt{ }$ & $\sqrt{ }$ & $\sqrt{ }$ & $\sqrt{ }$ & $\sqrt{ }$ & $\sqrt{ }$ \\
\hline
\end{tabular}

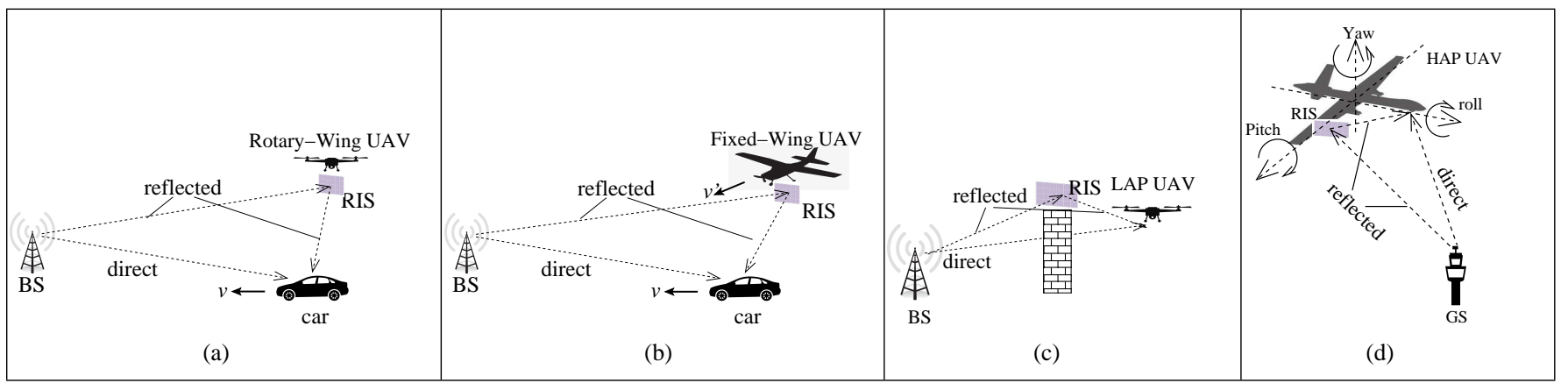

Fig. 1: Examples of unmanned aerial vehicles (UAV) scenarios.

Moreover, in this contribution, we focus our attention on the family of LoS-dominated UAV applications, as exemplied in Fig. 1, which will be elaborated on in Sec. II. In summary, the novel contributions of this work are:

- We construct a new analytical model of time-varying frequency-selective Ricean channels for high-dimensional and high-mobility RIS-assisted communication systems. The correlations of the fading channels in the SD, TD and FD imposed by the AoA/AoD, the Doppler and the OFDM operations, respectively, are carefully taken into account. The time-varying characteristics of the LoS and NLoS fading elements are analysed separately. Nonetheless, due to the tripple convolutions of the BSRIS links, RIS coefficients and RIS-User links, some of the LoS paths are entangled with the NLoS paths. The resultant effects imposed on the fading correlations are meticulously formulated.

- A low-complexity algorithm is proposed for RIS configuration based on the LoS characteristics. More explicitly, the LoS characteristics including the static information of initial coordinates, speed and carrier frequency, which can be used for predicting the LoS elements. Following this, the RIS coefficients are optimized for maximizing the predicted LoS power, hence they can be fed back in advance by a separate control link.

- We conceive a FD channel estimator for time-varying fading (FD-CE-TV). Owing to the fact that the RIS configuration is based on the deterministic LoS paths, the RIS-reflected links no longer have to be estimated one-by-one. Instead, the MMSE method is invoked in the FD in order to estimate the superimposed direct and reflected links, which is based on the pilot symbols that are periodically assigned in both the FD and the TD. Moreover, the ICI imposed by the LoS components is evaluated and eliminated from the received pilots, so that the FD signal detection is improved.

- In order to avoid ICI, we conceive furthermore a TD channel estimator for time-varying fading (TD-CE-TV). The pilot symbols are inserted in the TD only, where MMSE channel estimation is invoked in the TD in order to estimate the CIR taps one-by-one. Furthermore, in order to alleviate the ISI, successive interference cancellation (SIC) is invoked for TD signal detection. We note that for both FD-CE-TV and TD-CE-TV, the deployment of RIS is "transparant" to the transmitter, where the pilot pattern assignment in the FD and TD does not have to take into account the number of RIS-reflected links.

- Our simulation results demonstrate that the conventional ON/OFF and DFT based RIS channel estimation techniques [23], [26] conceived for time-invariant fading channels suffer from an irreducible error floor in the high-mobility scenarios, which is mitigated by the proposed MMSE schemes of FD-CE-TV and TD-CE-TV. Furthermore, our simulation results confirm that the RIS is capable of achieving substantial performance improvements both in terms of the unconstrained capacity of continuous-input continuous-output memoryless channels (CCMC), and the constrained capacity of discrete-input continuous-output memoryless channels (DCMC) as well as in terms of the bit error ratio (BER). Our simulation results are examined in six frequency bands licensed in $5 \mathrm{G}$, which confirms that the employment of RIS is especially beneficial in the lower $5 \mathrm{G}$ frequency bands.

The rest of this paper is organized as follows. The modelling of RIS assisted OFDM operating in doubly selective Ricean channels is presented in Sec. II. The optimization of RIS reflecting coefficients is devised in Sec. III. The MMSE channel estimation schemes are proposed in the FD and TD in Sec. IV, where sophisticated interference mitigation techniques are conceived. Finally, our performance results and 
TABLE II: OFDM notations.

\begin{tabular}{|l|l|l|}
\hline & Time-Domain (TD) & Frequency-Domain (FD) \\
\hline Transmitter & $\widetilde{s}_{i, n}$ & $s_{i, k}$ \\
\hline Fading & $\widetilde{h}_{i, n, l}$ & $h_{i, k}$ \\
\hline Receiver & $\widetilde{y}_{i, n}$ & $y_{i, k}$ \\
\hline
\end{tabular}

conclusions are offered in Secs. V and VI, respectively.

The following notations are used throughout the paper. The operations $(\cdot)^{*}$ and $(\cdot)^{H}$ denote the conjugate of a complex number and the Hermitian transpose of a complex matrix, respectively. The notations $\ln (\cdot)$ and $\exp (\cdot)$ refer to natural logarithm and natural exponential functions, respectively. The notations $p(\cdot)$ and $\mathrm{E}(\cdot)$ represent the probability and the expectation, respectively. $[\mathbf{A}]_{a, b}$ refers to the element on the $a$-th row and $b$-th column of matrix, and $\mathbf{A} \in \mathbb{C}^{c \times d}$ denotes that $\mathbf{A}$ is a complex-valued matrix of size $(c \times d)$.

\section{Modelling of RIS Assisted OFDM in Doubly SELECTIVE RiCEAN CHANNELS}

\section{A. General Setups}

We consider a downlink scenario where the coverage of a high-mobility user is enhanced by a RIS. In this paper, a single antenna is used both at the BS and at the user, which minimizes the CSI estimation overhead in the high-mobility scenarios considered. We note that as the numbers of antennas is increased at the BS and/or the user, the channel estimation scheme conceived in this paper can be directly applied using an ON/OFF pattern of antennas. However, this results in an excessive pilot overhead for the high-dimensional RIS link that grows with the number of antennas, which is not preferred in the high-mobility scenarios considered in this paper.

In this treatise, we focus our attention on the family of LoS-dominated UAV applications, as exemplied in Fig. 1. In more detail, in Fig. 1(a), a passive RIS covers the body of a rotary-wing UAV that remains stationary hovering in the air in order to assist a vehicle moving on the ground. By contrast, in Fig. 1(b), a RIS is deployed at a fixed-wing UAV that moves in the same direction as the vehicle travelling on the ground, so that a low Doppler is achieved for the critical RIS-User links. Moreover, in Fig. 1(c), a RIS is placed at a building top in order to assist a low-altitude UAV in the face of terrain shadowing, where the direct link is blocked by obstacles. Furthermore, in Fig. 1(d), the three-axis maneuvers of pitch, roll and yaw may lead to detrimental airframe shadowing for high-altitude UAVs, where the direct link is blocked by the body of the aircraft. This is highly hazardous, as the blockage may last as long as 74 seconds for a mission-critical UAV [44], [51]. As a remedy, a RIS covers the wing of the UAV, where a LoS link is retained from the RIS to the ground station (GS).

For the OFDM representations, the TD and FD notations follow the generic rules exemplified in Table II. Furthermore, the key model components of our RIS-assisted high-mobility OFDM systems are summarized in Table III, which will be detailed in the following sections.

\section{B. Multi-Carrier Modulation}

For the $i$-th OFDM symbol, the $k$-th SC is modulated as PSK/QAM as $\left\{s_{i, k}\right\}_{k=0}^{N-1}$, and then the TD signals $\left\{\widetilde{s}_{i, n}\right\}_{n=0}^{N-1}$ are generated by invoking the inverse discrete fourier transform (IDFT) as $\left(\widetilde{s}_{i, n}=\frac{1}{\sqrt{N}} \sum_{k=0}^{N-1} s_{i, k} \omega_{N}^{n k}\right)$, where $N$ refers to the number of SCs, while we have $\omega_{N}=\exp \left(j \frac{2 \pi}{N}\right)$. The IDFT can be written in matrix form as $\left(\widetilde{\mathbf{s}}_{i}=\frac{1}{\sqrt{N}} \mathbf{F}_{N}^{H} \mathbf{s}_{i}\right)$, where we have the TD sample $\widetilde{\mathbf{s}}_{i}=\left[\widetilde{s}_{i, 0}, \cdots, \widetilde{s}_{i, N-1}\right]^{T} \in \mathbb{C}^{N \times 1}$, the FD OFDM symbol $\mathbf{s}=\left[s_{i, 0}, \cdots, s_{i, N-1}\right]^{T} \in \mathbb{C}^{N \times 1}$ and the DFT matrix $\left[\mathbf{F}_{N}\right]_{a, b}=\exp \left(-j \frac{2 \pi}{N} a b\right)$ for $\mathbf{F}_{N} \in \mathbb{C}^{N \times N}$. A cyclic prefix of length $N_{c p}$ is attached to each TD OFDM symbol before its transmission.

\section{The Direct BS-User Link}

The PL of the direct BS-User link is given by [1]-[4], [40], [52], [53]:

$$
\begin{aligned}
\mathrm{PL}^{\mathrm{BS}-\text { User }} & =-10 \gamma^{\mathrm{BS}-\text { User }} \log _{10}\left(d^{\mathrm{BS}-\text { User }}\right)-20 \log _{10}\left(\frac{4 \pi}{\lambda}\right)+G_{e}^{\mathrm{BS}}+G_{e}^{\mathrm{User}} \\
& =10 \log _{10} \Gamma_{i, n}^{\mathrm{BS}-\text { User }} \mathrm{dB}
\end{aligned}
$$

where $\gamma^{\text {BS-User }}$ denotes the PL exponent of the BS-User link. The effective antenna gains are formulated as $\left(G_{e}^{\mathrm{BS}}=\right.$ $\left.\frac{4 \pi A_{e}^{\mathrm{BS}}}{\lambda^{2}}\right)$ and $\left(G_{e}^{\mathrm{User}}=\frac{4 \pi A^{\text {User }}}{\lambda^{2}}\right)$, where $A_{e}^{\mathrm{BS}}$ and $A_{e}^{\text {User }}$ are the antenna aperture of the BS and the user, respectively, while $f_{c}$ and $\lambda=\frac{c}{f_{c}}$ refer to the carrier frequency and the wavelength, respectively. The distance between the BS and user is updated for each sampling period according to $\left[d^{\mathrm{BS}-\text { User }}=\sqrt{\left(x_{\mathrm{BS}}-x_{\text {User }}\right)^{2}+\left(y_{\mathrm{BS}}-y_{\text {User }}\right)^{2}+\left(z_{\mathrm{BS}}-z_{\text {User }}\right)^{2}}\right.$, where $\left(x_{\mathrm{BS}}, y_{\mathrm{BS}}, z_{\mathrm{BS}}\right)$ and $\left(x_{\mathrm{User}}, y_{\mathrm{User}}, z_{\mathrm{User}}\right)$ refer to the coordinates of the BS and that of the user, respectively. The nonlogarithmic PL is given by $\Gamma_{i, n}^{\mathrm{BS}-\text { User }}$. In summary, the PL of (1) is a function of distance and carrier frequency/wavelength, which allows us to examine the performance of the RIS assisted highmobility systems at different frequency bands. On one hand, based on Friis' Law, the PL component of $\left[20 \log _{10}(4 \pi / \lambda)\right]$ increases quadratically with $f_{c}$. On the other hand, the antenna apertures $A_{e}^{\mathrm{BS}}$ and $A_{e}^{\text {User }}$ shrink as $f_{c}$ increases. As a result, given fixed antenna sizes, the effective antenna gains $G_{e}^{\mathrm{BS}}$ and $G_{e}^{\text {User }}$ also increase quadratically with $f_{c}$. More details on these relationships can be found in [40], [52], [53].

The BS-User link has $N_{h}^{\text {BS-User }}$ CIR taps, where the first tap represents the LoS, while the rest are NLoS paths. The LoS and NLoS power ratio is defined by the Ricean factor of $K^{\mathrm{BS}-\text { User }}$. The LoS tap of the $n$-th TD sampling period within the $i$-th OFDM symbol is given by:

$$
\widetilde{h}_{i, n, 0}^{\mathrm{BS}-\text { User }}=\sqrt{\frac{K^{\mathrm{BS}-\text { User }} \Gamma_{i, n}^{\mathrm{BS}-\text { User }}}{K^{\mathrm{BS}-\text {-ser }}+1}} \exp \left\{j 2 \pi\left[i\left(N+N_{c p}\right)+n\right] \Delta f_{i, n}^{\mathrm{BS}-\text { User,LoS }}\right\},
$$

where the LoS frequency offset imposed by the movement of the user is expressed as:

$$
\Delta f_{i, n}^{\mathrm{BS}-\text { User,LoS }}=\frac{f_{D}^{\mathrm{BS}-\text { User }} T}{N} \cos \left(\phi_{0}^{\mathrm{BS}-\text { User }}\right) .
$$

More explicitly, the maximum Doppler frequency of the BSUser link is given by $\left(f_{D}^{\mathrm{BS} \text {-User }}=\frac{v f_{c}}{d}\right)$, where $v$ and $c$ represent the user's velocity and the speed of light, respectively. The angle between the LoS and the direction of movement is evaluated by $\left(\phi_{0}^{\mathrm{BS}-\text { User }}=\arctan \frac{y_{\mathrm{BS}}-y_{\text {User }}}{x_{\mathrm{BS}}-x_{U \text { erer }}}\right)$. We note that both the path loss $\Gamma_{i, n}^{\mathrm{BS}}$ and AoA $\phi_{0}^{\mathrm{BS}}$ are updated based on the coordinates $\left(x_{\mathrm{BS}}, y_{\mathrm{BS}}, z_{\mathrm{BS}}\right)$ and $\left(x_{\mathrm{User}}, y_{\mathrm{User}}, z_{\mathrm{User}}\right)$ for each TD sampling period. Moreover, the NLoS taps of the BS-User link are generated by Clarke's model [54], [55] according to the complex normal distribution as:

$$
\widetilde{h}_{i, n, l}^{\mathrm{BS} \text {-User }} \in \mathcal{C} \mathcal{N}\left(0, \frac{\Gamma_{i, n}^{\mathrm{BS}-\text { User }}}{\left(K^{\mathrm{BS}-\text { User }}+1\right)\left(N_{h}^{\mathrm{BS}-\text { User }}-1\right)}\right),
$$




\begin{tabular}{|c|c|c|c|}
\hline & BS-User Link & BS-RIS Link & RIS-user Link \\
\hline Distance & $\begin{array}{l}d^{\text {BS-User }}= \\
\sqrt{\left(x_{\mathrm{BS}}-x_{\text {User }}\right)^{2}+\left(y_{\mathrm{BS}}-y_{\text {User }}\right)^{2}+\left(z_{\mathrm{BS}}-z_{\text {User }}\right)^{2}}\end{array}$ & $\begin{array}{l}d^{\mathrm{BS}-\mathrm{RIS}}= \\
\sqrt{\left(x_{\mathrm{BS}}-x_{\mathrm{RIS}}\right)^{2}+\left(y_{\mathrm{BS}}-y_{\mathrm{RIS}}\right)^{2}+\left(z_{\mathrm{BS}}-z_{\mathrm{RIS}}\right)^{2}}\end{array}$ & $\begin{array}{l}d^{\text {RIS-User }}= \\
\sqrt{\left(x_{\mathrm{RIS}}-x_{\text {User }}\right)^{2}+\left(y_{\mathrm{RIS}}-y_{\text {User }}\right)^{2}+\left(z_{\mathrm{RIS}}-z_{\text {User }}\right)^{2}}\end{array}$ \\
\hline $\begin{array}{l}\text { Path loss } \\
\text { exponent }\end{array}$ & $\gamma^{\text {BS-User }}$ & $\gamma^{\mathrm{BS}-\mathrm{RIS}}$ & $\gamma^{\text {RIS-User }}$ \\
\hline Path loss & $\Gamma_{i, n}^{\mathrm{BS}-\text { User }}=\left(d^{\mathrm{BS}-\text { User }}\right)^{-\gamma^{\mathrm{BS}-\mathrm{User}}}\left(\frac{4 \pi}{\lambda}\right)^{-2} 10^{0.1\left(G_{e}^{\mathrm{TX}}+G_{e}^{\mathrm{Rx}}\right)}$ & $\Gamma_{i, n}^{\mathrm{BS}-\mathrm{RIS}}=\left(d^{\mathrm{BS}-\mathrm{RIS}}\right)^{-\gamma^{\mathrm{BS}-\mathrm{RIS}}}\left(\frac{4 \pi}{\lambda}\right)^{-2} 10^{0.1 G_{e}^{\mathrm{TX}}}$ & $\Gamma_{i, n}^{\mathrm{RIS}-\text { User }}=\left(d^{\mathrm{RIS}-\text { User }}\right)^{-\gamma^{\mathrm{RIS}-\text { User }}}\left(\frac{4 \pi}{\lambda}\right)^{-2} 10^{0.1 G_{e}^{\mathrm{RX}}}$ \\
\hline $\begin{array}{l}\text { Ricean fac- } \\
\text { tor }\end{array}$ & $K^{\mathrm{BS}-\text { User }}$ & $K^{\text {BS-RIS }}$ & $K^{\text {RIS-User }}$ \\
\hline CIR taps & $N_{h}^{\text {BS-User }}$ & $N_{h}^{\mathrm{BS}-\mathrm{RIS}}$ & $N_{h}^{\text {RIS-User }}$ \\
\hline LoS power & $\frac{K^{\mathrm{BS}-\mathrm{User}} \Gamma_{i, n}^{\mathrm{BS}-\mathrm{User}}}{K^{\mathrm{BS}-\text { User }}+1}$ & $\frac{K^{\mathrm{BS}-\mathrm{RIS}} \Gamma_{i, n}^{\mathrm{BS}-\mathrm{RIS}}}{K^{\mathrm{BS}-\mathrm{RIS}}+1}$ & $\frac{K^{\text {RIS-User }} \Gamma_{i, n}^{\text {RIS-User }}}{K^{\text {RIS-User }}+1}$ \\
\hline $\begin{array}{l}\text { Max. } \\
\text { Doppler }\end{array}$ & $f_{D}^{\mathrm{BS}-\text { User }}$ & $f_{D}^{\mathrm{BS}-\mathrm{RIS}}$ & $f_{D}^{\mathrm{RIS}-\text { User }}$ \\
\hline LoS AoA & $\phi_{0}^{\mathrm{BS}-\text { User }}=\arctan \frac{y_{\mathrm{BS}}-y_{\text {User }}}{x_{\mathrm{BS}}-x_{\text {User }}}$ & $\phi_{0}^{\mathrm{BS}-\mathrm{RIS}}=\arctan \frac{y_{\mathrm{BS}}-y_{\mathrm{RIS}}}{x_{\mathrm{BS}}-x_{\mathrm{RIS}}}$ & $\phi_{0}^{\text {RIS-User }}=\arctan \frac{y_{\text {RIS }}-y_{\text {User }}}{x_{\text {RIS }}-x_{\text {User }}}$ \\
\hline RIS AoA & & 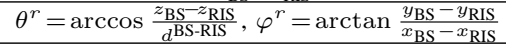 & \\
\hline RIS AoD & & & 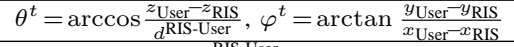 \\
\hline LoS offset & $\Delta f_{i, n}^{\mathrm{BS}-\text { User,LoS }}=\frac{f_{D}^{\mathrm{BS}-\text { User }} T}{N} \cos \left(\phi_{0}^{\mathrm{BS}-\text { User }}\right)$ & $\Delta f_{i, n}^{\mathrm{BS}-\mathrm{RIS}, \mathrm{LoS}}=\frac{f_{D}^{\mathrm{BS}-\mathrm{RIS}} T}{N} \cos \left(\phi_{0}^{\mathrm{BS}-\mathrm{RIS}}\right)$ & $\Delta f_{i, n}^{\mathrm{RIS}-U s e r, \mathrm{LoS}}=\frac{f_{D}^{\mathrm{RIS}-\mathrm{User}} T}{N} \cos \left(\phi_{0}^{\mathrm{RIS}-\mathrm{User}}\right)$ \\
\hline $\begin{array}{l}\text { NLoS } \\
\text { power }\end{array}$ & $\frac{\Gamma_{i, n}^{\text {BSUser }}}{\left(K^{\text {BS-User }}+1\right)\left(N_{h}^{\text {BS-User }}-1\right)}$ & $\frac{\Gamma_{i, n}^{\text {BS-RIS }}}{\left(K^{\text {BS-RIS }}+1\right)\left(N_{h}^{\text {BS-RIS }}-1\right)}$ & $\frac{\Gamma_{i, n}^{\text {RIS-User }}}{\left(K^{\text {RIS-User }}+1\right)\left(N_{h}^{\text {RIS-User }}-1\right)}$ \\
\hline $\begin{array}{l}\text { NLoS } \\
\text { auto- } \\
\text { correlation }\end{array}$ & 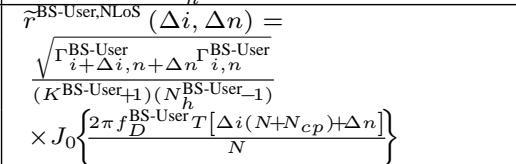 & $\begin{array}{l}\widetilde{r}^{\mathrm{BS}-\mathrm{RIS}, \mathrm{NLoS}}(\Delta i, \Delta n) \\
\sqrt{\Gamma_{i+\Delta i, n+\Delta n^{\mathrm{BS}} \Gamma_{i, n}^{\mathrm{BS}-\mathrm{RIS}}}^{\mathrm{BSI}}} \\
\left.\quad \times K^{\mathrm{BS}-\mathrm{RIS}}+1\right)\left(N_{h}^{\mathrm{BS}-R I S}-1\right) \\
\left.\quad \times \frac{2 \pi f_{D}^{\mathrm{BS}-\mathrm{RIS}} T\left[\Delta i\left(N+N_{c p}\right)+\Delta n\right]}{N}\right\}\end{array}$ & $\begin{array}{l}\widetilde{r}^{\text {RIS-User,NLoS }}(\Delta i, \Delta n)= \\
\sqrt{\Gamma_{i+\Delta i, n+\Delta n^{\text {RIS-User }} \Gamma_{i, n}^{\text {RIS-User }}}^{\text {Rer }}} \\
\quad \times J_{0}\left\{\frac{2 \pi f_{D}^{\text {RIS-User }} T\left[\Delta i\left(N+N_{c p}\right)+\Delta n\right]}{N}\right\}\end{array}$ \\
\hline
\end{tabular}

for $\left(1 \leq l \leq N_{h}^{\mathrm{BS}-\mathrm{User}}-1\right)$. The correlation over time is given by

$$
\begin{aligned}
& \widetilde{r}^{\mathrm{BS}-\text { User,NLoS }}(\Delta i, \Delta n)=E\left[\widetilde{h}_{i+\Delta i, n+\Delta n, l}^{\mathrm{BS}-\mathrm{User}}\left(\widetilde{h}_{i, n, l}^{\mathrm{BS}-\text { User }}\right)^{*}\right] \\
& =\frac{\sqrt{\Gamma_{i+\Delta \Delta, n+\Delta n}^{\mathrm{BS}-U \mathrm{U} e r} \Gamma_{i, n}^{\mathrm{BS}-\text { User }}}}{\left(K^{\mathrm{BS}-\text { User }}+1\right)\left(N_{h}^{\mathrm{BS}-\text { User }}-1\right)} J_{0}\left\{\frac{2 \pi f_{D}^{\mathrm{BS}-\text { User }} T\left[\Delta i\left(N+N_{c p}\right)+\Delta n\right]}{N}\right\},
\end{aligned}
$$

where $J_{0}(\cdot)$ is the Bessel function of the first kind.

\section{The BS-RIS Links}

The PL of the BS-RIS link is given by:

$$
\begin{aligned}
\mathrm{PL}^{\mathrm{BS}-\mathrm{RIS}} & =-10 \gamma^{\mathrm{BS}-\mathrm{RIS}} \log _{10}\left(d^{\mathrm{BS}-\mathrm{RIS}}\right)-20 \log _{10}\left(\frac{4 \pi}{\lambda}\right)+G_{e}^{\mathrm{BS}} \\
& =10 \log _{10} \Gamma_{i, n}^{\mathrm{BS}-\mathrm{RIS}} \mathrm{dB}
\end{aligned}
$$

where $\gamma^{\text {BS-RIS }}$ denotes the PL exponent of the BS-RIS link, while the distance between the BS and RIS is updated as $\left[d^{\mathrm{BS}-\mathrm{RIS}}=\sqrt{\left(x_{\mathrm{BS}}-x_{R I S}\right)^{2}+\left(y_{\mathrm{BS}}-y_{\mathrm{RIS}}\right)^{2}+\left(z_{\mathrm{BS}}-z_{\mathrm{RIS}}\right)^{2}}\right]$. The non-logarithmic PL is given by $\Gamma_{i, n}^{\mathrm{BS}-\mathrm{RIS}}$. We note that there is no antenna gain at the passive RIS.

The RIS is assumed to be comprised of $M$ passive reflecting elements, where a uniform planar array (UPA) is adopted. As a result, the LoS tap of each BS-RIS link associated with the $m$-th RIS element is modelled as:

$$
\begin{gathered}
\widetilde{h}_{i, n, 0}^{\mathrm{BS}-\mathrm{RIS}}=\sqrt{\frac{K^{\mathrm{BS}-\mathrm{RIS}} \Gamma_{i, n}^{\mathrm{BS}-\mathrm{RIS}}}{K^{\mathrm{BS}-\mathrm{RIS}}+1}} \exp \left\{j 2 \pi\left[i\left(N+N_{c p}\right)+n\right] \Delta f_{i, n}^{\mathrm{BS}-\mathrm{RIS}, \mathrm{LoS}}\right\} \\
\times\left[\mathbf{a}_{r}\left(\theta^{r}, \varphi^{r}\right)\right]_{m},
\end{gathered}
$$

for $(0 \leq m \leq M-1)$. The azimuth and elevation AoAs are evaluated based on the 3 -D coordinates as $\left(\theta^{r}=\arccos \frac{z_{\mathrm{BS}}-z_{\mathrm{RIS}}}{d^{\mathrm{BS}-\mathrm{RIS}}}\right)$ and $\left(\varphi^{r}=\arctan \frac{y_{\mathrm{BS}}-y_{\mathrm{RIS}}}{x_{\mathrm{BS}}-x_{\mathrm{RIS}}}\right)$, respectively. For the UPA having $M_{y}$ and $M_{z}$ elements on the $y$ and $z$ axes, the array response vector is given by:

$$
\begin{gathered}
\operatorname{auPA}_{\mathrm{UPA}}(\theta, \varphi)=\left[1, \cdots, \exp \left\{j \frac{2 \pi}{\lambda} d\left[m_{y} \sin \theta \cos \varphi+m_{z} \sin \varphi\right]\right\}\right. \\
\left.\quad \cdots, \exp \left\{j \frac{2 \pi}{\lambda} d\left[\left(M_{y}-1\right) \sin \theta \cos \varphi+\left(M_{z}-1\right) \sin \varphi\right]\right\}\right]^{T},
\end{gathered}
$$

where we have $\left[1 \leq m_{y} \leq\left(M_{y}-1\right)\right]$, $\left[1 \leq m_{z} \leq\left(M_{z}-1\right)\right]$ and $\left(M=M_{y} M_{z}\right)$, while $\left(d=\frac{\lambda}{2}\right)$ denotes the antenna spacing. The NLoS taps of the BS-RIS links are also generated by Clarke's model [54], [55] according to the complex normal distribution:

$$
\widetilde{h}_{i, n, l}^{\mathrm{BS}-\mathrm{RIS}} \in \mathcal{C} \mathcal{N}\left(0, \frac{\Gamma_{i, n}^{\mathrm{BS}-\mathrm{RIS}}}{\left(K^{\mathrm{BS}-\mathrm{RIS}}+1\right)\left(N_{h}^{\mathrm{BS}-\mathrm{RIS}}-1\right)}\right),
$$

for $\left(1 \leq l \leq N_{h}^{\mathrm{BS}-\mathrm{RIS}}-1\right)$ and $(0 \leq m \leq M-1)$. The associated TD correlation is given by:

$$
\begin{aligned}
\left.\widetilde{r}^{\mathrm{BS}-\mathrm{RIS}, \mathrm{NL} o \mathrm{~S}}(\Delta i, \Delta n)=E\left[\widetilde{h}_{i+\Delta i, n+\Delta n, l}^{\mathrm{BS}-\mathrm{RIS}}{ }_{m}\left(\widetilde{h}_{i, n, l}^{\mathrm{BS}-\mathrm{RIS}}\right)_{m}\right)^{*}\right] \\
=\frac{\sqrt{\Gamma_{i+\Delta, n+\Delta n}^{\mathrm{BS}-\mathrm{RIS}} \Gamma_{i, n}^{\mathrm{BS}-\mathrm{RIS}}}}{\left(K^{\mathrm{BS}-\mathrm{RIS}}+1\right)\left(N_{h}^{\mathrm{BS}-\mathrm{RIS}}-1\right)} J_{0}\left\{\frac{2 \pi f_{D}^{\mathrm{BS}-\mathrm{RIS}} T\left[\Delta i\left(N+N_{c p}\right)+\Delta n\right]}{N}\right\} .
\end{aligned}
$$

\section{E. The RIS-User Links}

The PL of the RIS-User link is formulated as:

$$
\begin{aligned}
& \mathrm{PL}^{\text {RIS-User }}=-10 \gamma^{\text {RIS-User }} \log _{10}\left(d^{\text {RIS-User }}\right)-20 \log _{10}\left(\frac{4 \pi}{\lambda}\right)+G_{e}^{\text {User }} \\
& \quad=10 \log _{10} \Gamma_{i, n}^{\text {RIS-User }} \mathrm{dB},
\end{aligned}
$$

where $\gamma^{\text {RIS-User }}$ denotes the PL exponent of the RIS-User link, while the distance between the relay and destination is updated as $\left[d^{\text {RIS-User }}=\sqrt{\left(x_{\mathrm{RIS}}-x_{\text {User }}\right)^{2}+\left(y_{\mathrm{RIS}}-y_{\text {User }}\right)^{2}+\left(z_{\mathrm{RIS}}-z_{\text {User }}\right)^{2}}\right]$. The non-logarithmic PL is given by $\Gamma_{i, n}^{\mathrm{RIS}-U s e r}$. Furthermore, the LoS tap of each RIS-User link associated with the $m$-th RIS element is modelled as:

$$
\begin{aligned}
& \widetilde{h}_{i, n, 0}^{\text {RIS-User } m}=\sqrt{\frac{K^{\text {RIS-User }} \Gamma_{i, n}^{\text {RIS-User }}}{K^{\text {RIS-User }}+1}} \exp \left\{j 2 \pi\left[i\left(N+N_{c p}\right)+n\right] \Delta f_{i, n}^{\text {RIS-User,LoS }}\right\} \\
& \times\left[\mathbf{a}_{t}\left(\theta^{t}, \varphi^{t}\right)\right]_{m},
\end{aligned}
$$

for $(0 \leq m \leq M-1)$. The UPA response vector is given by (8). The azimuth and elevation AoDs are evaluated as $\left(\theta^{t}=\arccos \frac{z_{\text {User }}-z_{\text {RIS }}}{d^{\text {RIS-User }}}\right)$ and $\left(\varphi^{t}=\arctan \frac{y_{\text {User }}-y_{\text {RIS }}}{x_{\text {User }}-x_{\text {RIS }}}\right)$, respectively. The NLoS taps of the RIS-User link $\left\{\widetilde{h}_{i, n, l}^{\mathrm{RIS}-\mathrm{User}_{m}}\right\}_{m=0}^{M-1}$ are also Guassian distributed in the same form as (9), and the TD correlation is in the same form as (10), where the power and Doppler are replaced by $\frac{\Gamma_{i, n}^{\text {RIS-User }}}{\left(K^{\text {RIS-User }}+1\right)\left(N_{h}^{\text {RIS-User }}-1\right)}$ and $f_{D}^{\text {RIS-User }}$, respectively.

\section{F. Received Signals Modelling}

For the cascaded BS-RIS-User reflected links, the transmitted TD signal is convolved three times with the BS-RIS link, as well as with the RIS reflecting coefficients and then with the RIS-User link. More explicitly, the TD signal arriving at the $m$-th RIS element is given by:

$$
\widetilde{y}_{i, n}^{\mathrm{BS}^{\mathrm{RIS}} \mathrm{m}_{m}}=\sum_{l_{1}=0}^{N_{h}^{\mathrm{BS}-\mathrm{RIS}}-1} \widetilde{h}_{i, n, l_{1}}^{\mathrm{BS}-\mathrm{RIS}} \widetilde{s}_{i, n-l_{1}} .
$$


Then the three-time convolution associated with the $m$-th RIS element is expressed as:

$$
\begin{aligned}
& \widetilde{y}_{i, n}^{\text {BS-RIS-User }_{m}}=\sum_{l_{2}=0}^{N_{h}^{\text {RIS-User }}-1} \widetilde{h}_{i, n, l_{2}}^{\text {RIS-Use }_{m}} \alpha_{i, n-l_{2}}^{m} \widetilde{y}_{i, n-l_{2}}^{\text {BS-RIS }_{m}} \\
& =\sum_{l_{1}=0}^{N_{h}^{\text {BS-RIS }}-1} \sum_{l_{2}=0}^{N_{h}^{\text {RIS-User }}-1} \alpha_{i, n-l_{2}}^{m} \widetilde{h}_{i, n-l_{2}, l_{1}}^{\text {BS-RIS }} \widetilde{h}_{i, n, l_{2}}^{\text {RIS-User } m} \widetilde{s}_{i, n-l_{1}-l_{2}} \\
& =\sum_{l=0}^{N_{h}^{\text {BS-RIS-User }}-1} \widetilde{\bar{h}}_{i, n, l}^{\text {BS-RIS-User }}{ }_{m} \widetilde{s}_{i, n-l},
\end{aligned}
$$

where we have $\left(N_{h}^{\text {BS-RIS-User }}=N_{h}^{\text {BS-RIS }}+N_{h}^{\text {RIS-User }}-1\right)$ and the cascaded reflected fading is:

$$
\widetilde{\bar{h}}_{i, n, l}^{\text {BS-RIS-User }_{m}}=\sum_{\forall l_{1}+l_{2}=l} \alpha_{i, n-l_{2}}^{m} \widetilde{h}_{i, n-l_{2}, l_{1}}^{\text {BS-RIS }_{m}} \widetilde{h}_{i, n, l_{2}}^{\text {RIS-User }_{m}} .
$$

Following this, the direct BS-User link and the cascaded BS-RIS-User reflected links are superimposed at the receiver, yielding:

$$
\begin{aligned}
\widetilde{y}_{i, n}=\sum_{l=0}^{N_{h}^{\text {BS-User- }}-1} \widetilde{h}_{i, n, l}^{\text {BS-User }} \widetilde{s}_{i, n-l}+\sum_{m=0}^{M-1} \sum_{l=0}^{N_{h}^{\text {BS-RIS-User }-1}} \widetilde{\bar{h}}_{i, n, l}^{\text {BS-RIS-User }_{m}} \widetilde{s}_{i, n-l} \\
=\sum_{l=0}^{N_{h}-1} \widetilde{h}_{i, n, l}+\widetilde{s}_{i, n-l}+\widetilde{v}_{i, n},
\end{aligned}
$$

where we have $\left[N_{h}=\max \left(N_{h}^{\text {BS-User }}, N_{h}^{\text {BS-RIS-User }}\right)\right]$. The equivalent $\mathrm{CIR}$ is given by:

$\widetilde{h}_{i, n, l}=\left\{\begin{array}{l}\widetilde{h}_{i, n}^{\text {BS-User }}+\sum_{m-1}^{M-1} \widetilde{\bar{h}}_{i, n, l}^{\text {BS-RIS-User } m}, \quad 0 \leq l \leq \min \left(N_{h}^{\text {BS-User }}-1, N_{h}^{\text {BS-RIS-User }}-1\right), \\ \widetilde{h}_{i, n, l}^{\text {BS-User }}, N_{h}^{\text {BS-RIS-User }} \leq l \leq N_{h}-1, \text { if } N_{h}=N_{h}^{\text {BS-User }}, \\ \sum_{m=0}^{M-1} \widetilde{\bar{h}}_{i, n, l}^{\text {BS-RIS-User } m}, N_{h}^{\text {BS-User }} \leq l \leq N_{h}-1, \text { if } N_{h}=N_{h}^{\text {BS-RIS-User }} .\end{array}\right.$

\section{G. The Effect of Double Selectivity}

For the case of time-invariant fading, i.e. when the fading envelope remains near-constant over an OFDM symbol period $\left\{\widetilde{h}_{i, l}=\widetilde{h}_{i, n, l}\right\}_{n=0}^{N-1}$, the received TD signals of (16) can be represented in matrix form as $\left(\widetilde{\mathbf{y}}_{i}=\widetilde{\mathbf{H}}_{i} \widetilde{\mathbf{s}}_{i}+\widetilde{\mathbf{v}}_{i}\right)$, where we have $\widetilde{\mathbf{y}}_{i}=\left[\widetilde{y}_{i, 0}, \cdots, \widetilde{y}_{i, N-1}\right]^{T} \in \mathbb{C}^{N \times 1}$ and $\widetilde{\mathbf{v}}_{i}=\left[\widetilde{v}_{i, 0}, \cdots, \widetilde{v}_{i, N-1}\right]^{T} \in$ $\mathbb{C}^{N \times 1}$, while the $(N \times N)$ circulant CIR matrix $\widetilde{\mathbf{H}}_{i}$ is constituted by the $N_{h}$ time-invariant taps $\left\{\widetilde{h}_{i, l}\right\}_{l=0}^{N_{h}-1}$. Following this, the received TD signals are transformed into the FD by DFT as $\left(y_{i, k}=\frac{1}{\sqrt{N}} \sum_{n=0}^{N-1} \widetilde{y}_{i, n} \omega_{N}^{-k n}\right)$, which can be expressed in matrix form as $\left(\mathbf{y}_{i}=\frac{1}{\sqrt{N}} \mathbf{F}_{N} \widetilde{\mathbf{y}}_{i}=\frac{1}{N} \mathbf{F}_{N} \widetilde{\mathbf{H}}_{i} \mathbf{F}_{N}^{H} \mathbf{s}_{i}+\frac{1}{\sqrt{N}} \mathbf{F}_{N} \widetilde{\mathbf{v}}_{i}\right)$. Owing to the circulant CIR matrix structure of $\widetilde{\mathbf{H}}_{i}$, the CFR matrix $\left[\mathbf{H}_{i}=\frac{1}{N} \mathbf{F}_{N} \widetilde{\mathbf{H}}_{i} \mathbf{F}_{N}^{H}=\operatorname{diag}\left(\mathbf{h}_{i}\right)\right]$ is diagonal. This facilitates OFDM's single-tap FD equalization, where each SC is demodulated independently without encountering ICI.

However, in the face of time-selectivity, the CIR taps in each row of the circulant CIR matrix $\widetilde{\mathbf{H}}_{i}$ are time-variant. Consequently, $\left(\mathbf{H}_{i}=\frac{1}{N} \mathbf{F}_{N} \widetilde{\mathbf{H}}_{i} \mathbf{F}_{N}^{H}\right)$ is no longer diagonal, which destroys OFDM's SC orthogonality. More explicitly, the received TD signals $\widetilde{y}_{i, n}$ may be extended as:

$$
\begin{aligned}
\widetilde{y}_{i, n} & =\sum_{l=0}^{N_{h}-1} \widetilde{h}_{i, n, l} \widetilde{s}_{i,<n-l>N}+\widetilde{v}_{i, n} \\
& =\frac{1}{\sqrt{N}} \sum_{l=0}^{N_{h}-1} \widetilde{h}_{i, n, l} \sum_{k=0}^{N-1} s_{i, k} \omega_{N}^{(n-l) k}+\widetilde{v}_{i, n} \\
& =\frac{1}{\sqrt{N}} \sum_{k=0}^{N-1} s_{i, k} \omega_{N}^{n k} \sum_{l=0}^{N_{h}-1} \widetilde{h}_{i, n, l} \omega_{N}^{-l k}+\widetilde{v}_{i, n} \\
& =\frac{1}{\sqrt{N}} \sum_{k=0}^{N-1} s_{i, k} h_{i, n, k} \omega_{N}^{n k}+\widetilde{v}_{i, n},
\end{aligned}
$$

where the CFRs are $\left(h_{i, n, k}=\sum_{l=0}^{N_{h}-1} \widetilde{h}_{i, n, l} \omega_{N}^{-l k}\right)$. Following this, the received FD signals after DFT may be extended as:

$$
\begin{aligned}
& y_{i, k}=\frac{1}{\sqrt{N}} \sum_{n=0}^{N-1} \widetilde{y}_{i, n} \omega_{N}^{-n k} \\
& \quad=\frac{1}{N} \sum_{n=0}^{N-1} \sum_{k^{\prime}=0}^{N-1} s_{i, k^{\prime}} h_{i, n, k^{\prime}} \omega_{N}^{n k^{\prime}} \omega_{N}^{-n k}+v_{i, k} \\
& =s_{i, k}\left(\frac{1}{N} \sum_{n=0}^{N-1} h_{i, n, k}\right)+\frac{1}{N} \sum_{k^{\prime} \neq k} s_{i, k^{\prime}} \sum_{n=0}^{N-1} h_{i, n, k^{\prime}} \omega_{N}^{n\left(k^{\prime}-k\right)}+v_{i, k} \\
& =s_{i, k} h_{i, k}+\beta_{i, k}+v_{i, k},
\end{aligned}
$$

where the ICI power is bounded by $E\left(\left|\beta_{i, k}\right|\right) \leq \frac{p i^{2}}{3}\left(f_{D} T\right)^{2}[56]$, [57], which is relatively small when $\left(f_{D} T \leq 0.02\right)$ [58]. The exact ICI power will be evaluated in Sec. IV.

\section{Optimization OF RIS REFLECTION COEFFICIENTS}

The objective of RIS optimization is to find the reflection coefficients that maximize the fading channel power. As the fading channels vary within an OFDM symbol duration, the RIS reflection coefficients $\left\{\alpha_{i, n}^{m}\right\}_{m=0}^{M-1}$ also become timevarying. As a result, the semidefinite relaxation [23] and the strongest-CIR maximization [26] designed for block fading are no longer applicable.

It is readily demonstrated by (18) that maximizing the $N_{h}$ CIR powers $\left\{\left|\widetilde{h}_{i, n, l}\right|^{2}\right\}_{l=0}^{N_{h}-1}$ and maximizing the $N$ CFR powers $\left\{\left|h_{i, n, k}\right|^{2}\right\}_{k=0}^{N-1}$ are equivalent. Owing to the fact that the number of CIR taps $N_{h}$ is generally much lower than the number of OFDM SCs $N$, the channel power is more concentrated in the TD than in the FD, as suggested in [26]. As a result, the following optimization problem is formulated for RIS configuration:

$$
\begin{aligned}
& \max _{\left\{\alpha_{i, n}^{m}\right\}_{m=0}^{M-1}} \sum_{l=0}^{N_{h}-1}\left|\widetilde{h}_{i, n, l}\right|^{2}, \\
& \text { subject to }\left|\alpha_{i, n}^{m}\right|=1, \forall m=0, \cdots, M-1 .
\end{aligned}
$$

This problem is non-convex due to the constant modulus constraint. Moreover, the same set of RIS reflection coefficients $\left\{\alpha_{i, n}^{m}\right\}_{m=0}^{M-1}$ calculated for the $n$-th sampling period in the $i$-th OFDM symbol have to maximize a total number of $N_{h}$ CIR powers $\left\{\left|\widetilde{h}_{i, n, l}\right|^{2}\right\}_{l=0}^{N_{h}-1}$ in (20), which is computationally complex to solve. In order to conceive a low-complexity algorithm that is feasible for rapidly fluctuating fading channels, we seek the RIS reflection coefficients that maximize the dominant LoS tap, which is extended based on (17) as:

$$
\begin{aligned}
\widetilde{h}_{i, n, 0} & =\widetilde{h}_{i, n, 0}^{\text {BS-User }}+\sum_{m=0}^{M-1} \widetilde{\bar{h}}_{i, n, 0}^{\text {BS-RIS-User }_{m}} \\
& =\widetilde{h}_{i, n, 0}^{\text {BS-User }}+\sum_{m=0}^{M-1} \alpha_{i, n}^{m} \widetilde{h}_{i, n, 0}^{\text {BS-RIS }_{m}} \widetilde{h}_{i, n, 0}^{\text {RIS-User }_{m}} .
\end{aligned}
$$

The convolution operation

of $\left(\sum_{\forall l_{1}+l_{2}=l} \alpha_{i, n-l_{2}}^{m} \widetilde{h}_{i, n-l_{2}, l_{1}}^{\text {BS-RIS }_{m}} \widetilde{h}_{i, n, l_{2}}^{\text {RIS- }_{m}}\right)$ in (15) is avoided in the LoS representation of (21). As a result, the set $\left\{\alpha_{i, n}^{m}\right\}_{m=0}^{M-1}$ is only related to the fading elements associated with the same sampling index $n$ in (21). Based on this, the RIS reflection coefficients that maximize the dominant LoS power are given by:

$$
\alpha_{i, n}^{m}=\frac{\widetilde{h}_{i, n, 0}^{\text {BS-User }}\left(\widetilde{h}_{i, n, 0}^{\text {BS-RIS }} m \widetilde{h}_{i, n, 0}^{\text {RIS-User } m}\right)^{*}}{\left|\widetilde{h}_{i, n, 0}^{\text {BS-User }}\left(\widetilde{h}_{i, n, 0}^{\text {BS-RIS } m} \widetilde{h}_{i, n, 0}^{\text {RIS-User } m}\right)^{*}\right|} .
$$

Alternatively, (20) is suboptimally solved by the successive convex approximation (SCA) technique in the FD in [23], which obtains a stationary point at the cost of polynomial complexity in terms of $N$ and $M$. As a further advance, the non-convex optimization problem may be solved by deep reinforcement learning [59] without using sophisticate mathematical model-based optimization techniques. Nonetheless, the LoS-based solution of (22) and the SCA-based solution of [23] have neglible performance differences in LoS-dominated high-mobility UAV scenarios considered in this paper, which will be confirmed by our simulation results in Sec. V.

\section{MMSE CHANNEL ESTIMATION TECHNiQUES}

In this section, we conceive MMSE channel estimation methods that operates either in the FD or TD, which are referred to as FD-CE-TV and TD-CE-TV, respectively. The pilot assignment patterns of FD-CE-TV and TD-CE-TV are 


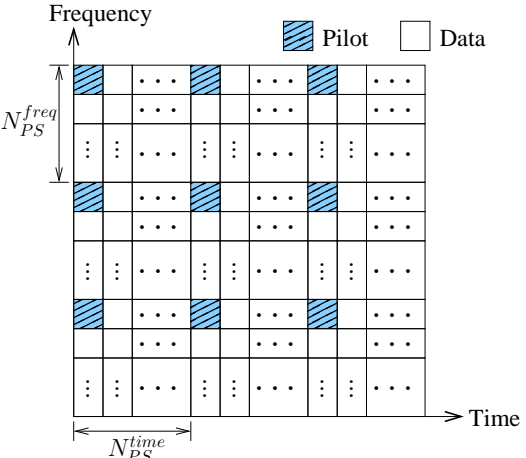

(a) FD-CE-TV

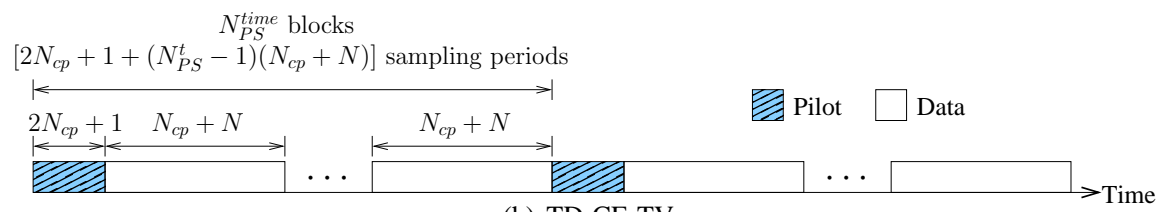

(b) TD-CE-TV

Fig. 2: Pilot patterns for frequency-domain channel estimation for time-varing fading (FD-CE-TV) and time-domain channel estimation for time-varing fading (TD-CE-TV).

portrayed in Fig. 2, which will be further elaborated on in the following sections.

\section{A. FD-CE-TV}

Owing to the fact that the determinstic LoS paths are used for RIS configuration, the channel estimator no longer has to estimate the direct and reflected links one-by-one. The most important objective is to formulate the correlation functions of RIS assisted OFDM in doubly selective fading, so that the MMSE channel estimates can be devised for signal detection. More explicitly, first of all, the LoS paths of the cascaded BS-RIS-User links of (15) may be extended as:

$$
\widetilde{\bar{h}}_{i, n, 0}^{\text {BS-RIS-User }_{m}}=\alpha_{i, n}^{m} \widetilde{h}_{i, n, 0}^{\text {BS-RIS }_{m}} \widetilde{h}_{i, n, 0}^{\text {RIS-User } m_{m}},
$$

while the NLoS paths are extended as:

$$
\begin{aligned}
\widetilde{\bar{h}}_{i, n, l}^{\text {BS-RIS-User }_{m}}= & \alpha_{i, n-l}^{m} \widetilde{h}_{i, n-l, 0}^{\text {BS-RIS }_{m}} \widetilde{h}_{i, n, l}^{\text {RIS-User }_{m}}+\alpha_{i, n}^{m} \widetilde{h}_{i, n, l}^{\text {BS-RIS }_{m}} \widetilde{h}_{i, n-\text { User }_{m}}^{\text {RIS }_{i, n}} \\
& +\sum_{\forall l_{1}+l_{2}=l, l_{1} \neq 0, l_{2} \neq 0} \alpha_{i, n-l_{2}}^{m} \widetilde{h}_{i, n-l_{2}, l_{1}}^{\text {BS-RI }_{m}} \widetilde{h}_{i, n, l_{2}}^{\text {RIS-User }_{m}}
\end{aligned}
$$

for $\left(l=1, \cdots, N_{h}-1\right)$. It can be seen in (24) that due to the tripple convolutions of the BS-RIS links, RIS coefficients and RIS-User links, some of the LoS paths are entangled with the NLoS paths, which has to be carefully taken into account. As a result, the correlation of the NLoS paths is given by:

$$
\begin{aligned}
& \widetilde{\bar{r}}_{l}^{\text {BS-RIS-User,NLoS }_{m}}(\Delta i, \Delta n)=E\left\{\begin{array}{l}
\widetilde{\bar{h}}_{i+\Delta i, n+\Delta n, l}^{\text {BS-RIS-User }_{m}}\left(\widetilde{\bar{h}}_{i, n, l}^{\text {BS-RIS-User }_{m}}\right)^{*}
\end{array}\right. \\
& =\alpha_{i+\Delta i, n+\Delta n-l}^{m} \widetilde{h}_{i+\Delta i, n+\Delta n-l, 0}^{\mathrm{BS}^{\mathrm{BIS}}{ }_{m}}\left(\alpha_{i, n-l}^{m} \widetilde{h}_{i, n-l, 0}^{\mathrm{BS}^{\mathrm{BSIS}}}\right) \stackrel{*}{\widetilde{r}^{\mathrm{RIS}-U s e r, N L o S}}(\Delta i, \Delta n)
\end{aligned}
$$

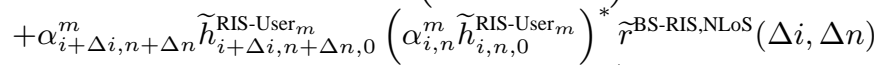

$$
\begin{aligned}
& +\sum_{\forall l_{1}+l_{2}=l, l_{1} \neq 0, l_{2} \neq 0} \alpha_{i+\Delta i, n+\Delta n-l_{2}}^{m}\left(\alpha_{i, n-l_{2}}^{m}\right)^{*} \\
& \times \widetilde{r}^{\text {RIS-User-NLoS }}(\Delta i, \Delta n) \widetilde{r}^{\text {BS-RIS,NLoS }}(\Delta i, \Delta n),
\end{aligned}
$$

for $\left(l=1, \cdots, N_{h}-1\right)$. Based on this, the TD correlation function of the NLoS of the superimposed direct and reflected links $\widetilde{h}_{i, n, l}$ of (17) is given by (26).

In the FD, the composite direct and reflected links $h_{i, k}$ seen in (19) may be extended as:

$$
\begin{aligned}
h_{i, k} & =\frac{1}{N} \sum_{n=0}^{N-1} h_{i, n, k} \\
& =\frac{1}{N} \sum_{n=0}^{N-1}\left(\widetilde{h}_{i, n, 0}+\sum_{l=1}^{N_{h}-1} \widetilde{h}_{i, n, l} \omega_{N}^{-l k}\right),
\end{aligned}
$$

where the LoS part is the same for all subcarriers:

$$
h_{i}^{\mathrm{LoS}}=\frac{1}{N} \sum_{n=0}^{N-1} \widetilde{h}_{i, n, 0},
$$

while the NLoS part is given by:

$$
h_{i, k}^{\mathrm{NLoS}}=\frac{1}{N} \sum_{n=0}^{N-1} \sum_{l=1}^{N_{h}-1} \widetilde{h}_{i, n, l} \omega_{N}^{-l k} .
$$

As a result, the FD correlation function of the NLoS of composite direct and reflected links $h_{i, k}^{\mathrm{NLoS}}$ is given by:

$$
\begin{aligned}
r(\Delta i, \Delta k)=E\left\{h_{i+\Delta i, k+\Delta k} h_{i, k}^{*}\right\} \\
=\frac{1}{N^{2}} E\left\{\left[\sum_{n_{1}=0}^{N-1} h_{i+\Delta i, n_{1}, k+\Delta k}\right]\left[\sum_{n_{2}=0}^{N-1} h_{i, n_{2}, k}^{*}\right]\right\} \\
=\frac{1}{N^{2}} E\left\{\left[\sum_{n_{1}=0}^{N-1} \sum_{l_{1}=0}^{N_{h}-1} \widetilde{h}_{i+\Delta i, n_{1}, l_{1}} w_{N}^{\left.-l_{1}(k+\Delta k)\right]}\right.\right. \\
\left.\quad \times\left[\sum_{n_{2}=0}^{N-1} \sum_{l_{2}=0}^{N_{h}-1} \widetilde{h}_{i, n_{2}, l_{2}}^{*} w_{N}^{l_{2} k}\right]\right\} \\
=\frac{1}{N^{2}} E\left\{\sum_{n_{1}=0}^{N-1} \sum_{n_{2}=0}^{N-1} \sum_{l=0}^{N_{h}-1} \widetilde{h}_{i+\Delta i, n_{1}, l} \widetilde{h}_{i, n_{2}, l}^{*} w_{N}^{-l \Delta k}\right\} \\
=r^{\operatorname{LoS}}(\Delta i)+r^{\operatorname{LoS}}(\Delta i, \Delta k),
\end{aligned}
$$

where the correlation functions of the LoS and NLoS components are given by:

$$
\begin{aligned}
& r^{\mathrm{LoS}}(\Delta i)=\frac{1}{N^{2}} \sum_{n_{1}=0}^{N-1} \sum_{n_{2}=0}^{N-1} \widetilde{h}_{i, n_{2}, 0} \widetilde{h}_{i, n_{2}, 0}^{*}, \\
& r^{\mathrm{NLoS}}(\Delta i, \Delta k)=\frac{1}{N^{2}} \sum_{n_{1}=0}^{N-1} \sum_{n_{2}=0}^{N-1} \sum_{l=1}^{N_{h}-1} \widetilde{r}_{l}^{\mathrm{NLoS}}(\Delta i, \Delta n) \omega_{N}^{-l \Delta k} .
\end{aligned}
$$

The pilot pattern of FD-CE-TV is portrayed in Fig. 2(a). As for the pilot OFDM symbol, a total of $N_{p}$ pilots are inserted into the FD with a pilot spacing of $N_{P S}^{f r e q}$, where we have $\left(N=N_{p} N_{P S}^{f r e q}\right)$. Based on (19), the received pilots in the FD are given by:

$$
y_{i_{u}, k_{u}}=h_{i_{u}, k_{u}}+\beta_{i_{u}, k_{u}}+v_{i_{u}, k_{u}},
$$

where $\left(i_{u}, k_{u}\right)$ indicates the position of the $u$-th pilot, as seen in Fig. 2(a). In order to evaluate the CFR $h_{i, k}$, the MMSE solution observes a total number of $N_{w}$ pilots in the FD and TD that precede $h_{i, k}$, and then produces the estimated $\hat{h}_{i, k}$ based on the correlation function of (30) as:

$$
\begin{aligned}
\hat{h}_{i, k} & =\sum_{u=0}^{N_{w}-1}\left(w_{i, k, u}\right)^{*} y_{i_{u}, k_{u}}=\left(\mathbf{w}_{i, k}\right)^{H} \check{\mathbf{y}}_{i, k} \\
& =\mathbf{e}_{i, k}\left(\mathbf{C}_{i, k}\right)^{-1} \check{\mathbf{y}}_{i, k},
\end{aligned}
$$

where we have the MMSE filter's inputs $\left[\check{\mathbf{y}}_{i, k}\right]_{u}=$ $y_{i_{u}, k_{u}}$ as well as the MMSE filter's correlations $\left[\mathbf{e}_{i, k}=\right.$ $\left.E\left\{h_{i, k}^{\mathrm{NLoS}}\left(\check{\mathbf{y}}_{i, k}\right)^{H}\right\}\right]_{u}=r^{\mathrm{LoS}}\left(i-i_{u}\right)+r^{\mathrm{NLoS}}\left(i-i_{u}, k-k_{u}\right)$ and $\left[\mathbf{C}_{i, k}=E\left\{\check{\mathbf{y}}_{i, k}\left(\check{\mathbf{y}}_{i, k}\right)^{H}\right\}\right]_{u_{1}, u_{2}}=r^{\mathrm{LoS}}\left(i_{u_{1}}-i_{u_{2}}\right)+r^{\mathrm{NLoS}}\left(i_{u_{1}}-\right.$ $\left.i_{u_{2}}, k_{u_{1}}-k_{u_{2}}\right)+\left(N_{0}\right)_{u_{1}=u_{2}}$, where $N_{0}$ is only added when $\left(u_{1}=u_{2}\right)$.

In high-Doppler scenarios, the CFRs vary more rapidly in the TD than in the FD. Therefore, it becomes more beneficial to dedicate the MMSE observation efforts to the TD of Fig. 2(a). For example, let us consider the case of having $(N=64) \mathrm{SCs}$ and $\left(N_{p}=4\right)$ pilots. Then the MMSE filters associated with $\left(N_{w}=8\right)$ can utilize 8 pilot positions $\left\{\left(i_{u}, k_{u}\right)\right\}_{u=0}^{N_{w}-1}$ spanning over 2 pilot OFDM symbols. By contrast, we propose to firstly invoke the MMSE method to 


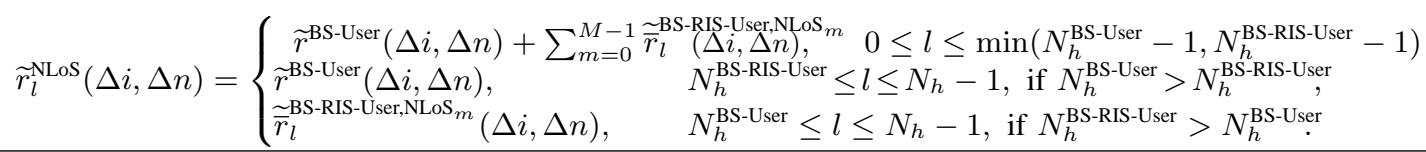

estimate the CFRs associated with the same SC indices as the pilots, which are indicated by $k \in\left\{0, N_{P S}^{f r e q}, 2 N_{P S}^{f r e q}, \cdots,\left(N_{p}-\right.\right.$ 1) $\left.N_{P S}^{f r e q}\right\}$. In this way, for each SC index $k$, the MMSE filter's $\left(N_{w}=8\right)$ input can utilize 8 pilot positions $\left\{\left(i_{u}, k\right)\right\}_{u=0}^{N_{w}-1}$ from 8 pilot OFDM symbols, which offer better observations in the TD. This first step follows a "horizontal comb" fashion in Fig. 2(a). Following this, we propose to complete all CFR estimation in a "vertical comb" fashion. More explicitly, upon obtaining a subset of CFRs $\hat{h}_{i, k}$ for the SC indices of $k \in\left\{0, N_{P S}^{f r e q}, 2 N_{P S}^{f r e q}, \cdots,\left(N_{p}-1\right) N_{P S}^{f r e q}\right\}$, which form a vector $\hat{\mathbf{h}}_{i}^{p} \in \mathbb{C}^{N_{p} \times 1}$, the equivalent CIRs of the $i$-th OFDM symbol period may be recovered by performing $N_{p}$-point IDFT as $\left[\hat{\tilde{\mathbf{h}}}_{i}^{p}\right]_{0:\left(N_{h}-1\right)}=\frac{\sqrt{N}}{N_{p}}\left[\mathbf{F}_{N_{p}}^{H} \hat{\mathbf{h}}_{i}^{p}\right]_{0:\left(N_{h}-1\right)}$, where only the first $N_{h}$ elements gleaned from the IDFT are retained in the $(N \times 1)$-element CIR vector $\hat{\widetilde{\mathbf{h}}}_{i}^{p}$, while the remaining $\left(N-N_{h}\right)$ elements in $\hat{\widetilde{\mathbf{h}}}_{i}^{p}$ are all zeros. Finally, the full $(N \times 1)$-element CFR vector of the $i$-th OFDM symbol is obtained by the $N$ point DFT as $\left(\hat{\mathbf{h}}_{i}=\frac{1}{\sqrt{N}} \mathbf{F}_{N} \hat{\tilde{\mathbf{h}}}_{i}^{p}\right)$.

Furthermore, in order to improve the MMSE performance, the LoS part of ICI can be eliminated from the received pilots. The overall ICI of the pilot symbols are given by:

$$
\begin{aligned}
& \beta_{i_{u}, k_{u}}=\frac{1}{N} \sum_{k^{\prime} \neq k_{u}, k^{\prime} \in \mathcal{P}} \sum_{n=0}^{N-1} h_{i_{u}, n, k^{\prime}} \omega_{N}^{n\left(k^{\prime}-k_{u}\right)} \\
& \quad=\frac{1}{N} \sum_{k^{\prime} \neq k_{u}, k^{\prime} \in \mathcal{P}} \sum_{n=0}^{N-1}\left(\widetilde{h}_{i_{u}, n, 0}+\sum_{l=1}^{N_{h}} \widetilde{h}_{i_{u}, n, l} \omega_{N}^{-l k^{\prime}}\right) \omega_{N}^{n\left(k^{\prime}-k_{u}\right)} \\
& =\beta_{i_{u}, k_{u}}^{\mathrm{LoS}}+\beta_{i_{u}, k_{u}}^{\mathrm{NLoS}},
\end{aligned}
$$

where the LoS and NLoS parts are formulated as:

$$
\begin{aligned}
& \beta_{i_{u}, k_{u}}^{\mathrm{LoS}}=\frac{1}{N} \sum_{k^{\prime} \neq k_{u}, k^{\prime} \in \mathcal{P}} \sum_{n=0}^{N-1} \widetilde{h}_{i_{u}, n, 0} \omega_{N}^{n\left(k^{\prime}-k_{u}\right)}, \\
& \beta_{i_{u}, k_{u}}^{\mathrm{NLoS}}=\frac{1}{N} \sum_{k^{\prime} \neq k_{u}, k^{\prime} \in \mathcal{P}} \sum_{n=0}^{N-1} \sum_{l=1}^{N_{h}} \widetilde{h}_{i_{u}, n, l} \omega_{N}^{n\left(k^{\prime}-k_{u}\right)-l k^{\prime}} .
\end{aligned}
$$

Therefore, the LoS part of ICI can be evaluated and subtracted from the received pilots as $\left[\check{\mathbf{y}}_{i, k}\right]_{u}=y_{i_{u}, k_{u}}-\beta_{i_{u}, k_{u}}^{\mathrm{LoS}}$.

Finally, upon obtaining all estimated CFRs, OFDM's classic single-tap FD equalization may be invoked based on the received signal model of (19) as $\hat{s}_{i, k}=\mathbb{M}^{-1}\left(y_{i, k} \hat{h}_{i, k}^{*} /\left|\hat{h}_{i, k}\right|^{2}\right)$ [4], [60].

\section{B. $T D-C E-T V$}

As seen in Fig. 2(b), the TD signal transmission is organized into frames. In the $u$-th frame, the first symbol is a pilot that takes up $\left(2 N_{c p}+1\right)$ sampling periods. Following the pilot, there are $\left(N_{P S}^{t i m e}-1\right)$ OFDM symbols, each of which lasts $\left(N+N_{c p}\right)$ sampling periods. The first $N_{c p}$ cyclic prefix periods are removed at the receiver. In summary, in each frame, there are $N_{P S}^{t i m e}$ symbols that last a total of $\left[N_{\text {frame }}=\left(2 N_{c p}+1\right)+\right.$ $\left.\left(N_{P S}^{t i m e}-1\right)\left(N+N_{c p}\right)\right]$ sampling periods. For the sake of clarity, the subscript of OFDM index $i$ is replaced by the superscripts of both frame index and symbol index. More explicitly, the pilots and data are represented by $\widetilde{s}_{n}^{u, 0}$ and $\widetilde{s}_{n}^{u, t}$ for the $(i=$ $\left.u N_{\text {frame }}+N_{c p}+n\right)$-th sampling period for the pilot and the $[i=$ $u N_{\text {frame }}+\left(2 N_{c p}+1\right)+(t-1)\left(N+N_{c p}\right)+n$ ]-th sampling period for the data, respectively, where we have $\left(t=1,2, \cdots, N_{P S}^{t i m e}-1\right)$.
In order to sample the $N_{h}$ CIR taps, the transmitted TD pilots are designed as:

$$
\widetilde{s}_{n}^{u, 0}=\left\{\begin{array}{ll}
1, & n=0 \\
0, & n=1,2, \cdots, N_{c p}
\end{array} .\right.
$$

As a result, the received TD pilots are given by:

$$
\widetilde{y}_{n}^{u, 0}= \begin{cases}\widetilde{h}_{l, l}^{u, 0}+\widetilde{v}_{l}^{u, 0}, & n=l=0,1, \cdots, N_{h} \\ \widetilde{v}_{n}^{u, 0}, & n=N_{h}+1, N_{h}+2, \cdots, N_{c p}\end{cases}
$$

Following this, the transmitted data OFDM samples in the TD are denoted by $\widetilde{s}_{n}^{u, t}$. The received signals in the TD are given by:

$$
\widetilde{y}_{n}^{u, t}=\sum_{l=0}^{N_{h}} \widetilde{h}_{n, l}^{u, t} \widetilde{s}_{<n-l>N}^{u, t}+\widetilde{v}_{n}^{u, t} .
$$

Based on this signal transmission pattern, the correlation between the CIRs on pilot positions is given by $E\left[\widetilde{h}_{l, l}^{u+\Delta u, 0}\left(\widetilde{h}_{l, l}^{u, 0}\right)^{*}\right]=\widetilde{r}^{\mathrm{NLoS}}\left(\Delta u N_{\text {frame }}, 0\right)$, and the correlation between CIRs on the pilot and data positions is given by $E\left[\widetilde{h}_{n, l}^{u+\Delta u, t}\left(\widetilde{h}_{l, l}^{u, 0}\right)^{*}\right]=\widetilde{r}^{\mathrm{NLoS}}\left(\Delta u N_{\text {frame }}+\left(2 N_{c p}+1\right)+(t-1)(N+\right.$ $\left.\left.N_{c p}\right), n-l\right)$. As a result, the MMSE solution of the estimated $\widetilde{h}_{n, l}^{u, t}$ is formulated as:

$$
\hat{\widetilde{h}}_{n, l}^{u, t}=\mathbf{e}_{n, l}^{u, t}\left(\mathbf{C}_{l}^{u}\right)^{-1} \check{\widetilde{\mathbf{y}}}_{l}^{u},
$$

where the $\left(N_{w} \times 1\right)$-element received pilot vector is given by $\check{\widetilde{y}}_{l}^{u}=\left[\widetilde{y}_{l}^{u-N_{w}+1,0}, \widetilde{y}_{l}^{u-N_{w}+2,0}, \cdots, \widetilde{y}_{l}^{u, 0}\right]^{T}$. Furthermore, the MMSE's correlation matrices are given by (40) and (41), where we have $\left[\Delta_{t}=\left(2 N_{c p}+1\right)+(t-1)\left(N+N_{c p}\right)\right]$.

Upon obtaining the estimated CIRs, the signal detection may also be performed in the TD in order to avoid the Dopplerdependent ICI. More explicitly, the TD received signal of (18) may be expressed in matrix form as shown in (42), where the frame index $u$ and symbol index $t$ are omitted for the sake of simplicity. The resultant MMSE detector that minimizes the ISI is given by:

$$
\mathbf{z}=\mathbf{G}_{\mathrm{MMSE}}^{H} \widetilde{\mathbf{y}},
$$

where the MMSE matrix is expressed as:

$$
\mathbf{G}_{\mathrm{MMSE}}^{H}=\left(\mathbf{H}^{H} \mathbf{H}+N_{0} \mathbf{I}_{N}\right)^{-1} \mathbf{H}^{H} .
$$

Then successive interference cancelation (SIC) can be invoked based on the signal-to-interference-and-noise-ratio (SINR):

$$
\eta_{k}=\frac{\left|\mathbf{g}_{k}^{H} \mathbf{h}_{k}\right|^{2}}{\sum_{k^{\prime} \neq k}\left|\mathbf{g}_{k^{\prime}}^{H} \mathbf{h}_{k^{\prime}}\right|^{2}+N_{0}\left\|\mathbf{g}_{k}\right\|^{2}},
$$

where $\mathbf{g}_{k}$ and $\mathbf{h}_{k}$ refer to the $k$-th column in $\mathbf{G}_{\text {MMSE }}$ and $\mathbf{H}$, respectively. As a result, the signals associated with higher SINR will be demodulated first and then substracted from $\tilde{\mathbf{y}}$. The pseudocode for the proposed SIC is provided in Table IV.

\section{Capacity Evaluations}

In this section, we evaluate the key system performance metrics of CCMC capacity and DCMC capacity in both the FD and TD. More explicitly, based on (19), the CCMC capacity is given by maximizing the mutual information $I(s ; y)$ between the channel's input and output signals over the distribution of $p(s)$ as $C^{\mathrm{CCMC}}(S N R)=\max _{p(s)} I(s ; y)=\max _{p(s)} H(y)-$ $H(y \mid s)$, where the entropies are maximized by Gaussian input 


$$
\begin{aligned}
& \begin{array}{l}
\mathbf{C}_{l}^{u}=\mathbf{R}_{y y}=E\left[\check{\tilde{\mathbf{y}}}_{l}^{u}\left(\check{\tilde{\mathbf{y}}}_{l}^{u}\right)^{H}\right]=N_{0} \mathbf{I}_{N_{w}}+ \\
{\left[\begin{array}{lllll}
\widetilde{r}^{\mathrm{NLoS}}(0,0) & \widetilde{r}^{\mathrm{NLoS}}\left(-N_{\text {frame }}, 0\right) & \widetilde{r}^{\mathrm{NLoS}}\left(-2 N_{\text {frame }}, 0\right) & \cdots & \widetilde{r}^{\mathrm{NLoS}}\left(-\left(N_{w}-1\right) N_{\text {frame }}, 0\right) \\
\widetilde{r}^{\mathrm{NLoS}}\left(N_{\text {frame }}, 0\right) & \widetilde{r}^{\mathrm{NLoS}}(0,0) & \widetilde{r}^{\mathrm{NLoS}}\left(-N_{\text {frame }}, 0\right) & \cdots & \widetilde{r}^{\mathrm{NLoS}}\left(-\left(N_{w}-2\right) N_{\text {frame }}, 0\right) \\
\widetilde{r}^{\mathrm{NLoS}}\left(2 N_{\text {frame }}, 0\right) & \widetilde{r}^{\mathrm{NLoS}}\left(N_{\text {frame }}, 0\right) & \widetilde{r}^{\mathrm{NLoS}}(0,0) & \cdots & \widetilde{r}^{\mathrm{NLoS}}\left(-\left(N_{w}-3\right) N_{\text {frame }}, 0\right) \\
\vdots & \vdots & \vdots & \ddots & \vdots \\
\tilde{\widetilde{r}}^{\mathrm{NLoS}}\left(\left(N_{w}-1\right) N_{\text {frame }}, 0\right) & \widetilde{r}^{\mathrm{NLoS}}\left(\left(N_{w}-2\right) N_{\text {frame }}, 0\right) & \widetilde{r}^{\mathrm{NLoS}}\left(\left(N_{w}-3\right) N_{\text {frame }}, 0\right) & \cdots & \widetilde{r}^{\mathrm{NLoS}}(0,0)
\end{array}\right] .}
\end{array} \\
& \mathbf{e}_{n, l}^{u, t}=\mathbf{R}_{h y}=E\left[\widetilde{h}_{n, l}^{u, t}\left(\check{\widetilde{\mathbf{y}}}_{l}^{u}\right)^{H}\right] \\
& =\left[\begin{array}{llllll}
\widetilde{r}^{\mathrm{NLoS}}\left(\left(N_{w}-1\right) N_{\text {frame }}+\Delta_{t}, n-l\right) & \widetilde{r}^{\mathrm{NLoS}}\left(\left(N_{w}-2\right) N_{\text {frame }}+\Delta_{t}, n-l\right) & \cdots & \widetilde{r}^{\mathrm{NLoS}}\left(\Delta_{t}, n-l\right)
\end{array}\right] .
\end{aligned}
$$

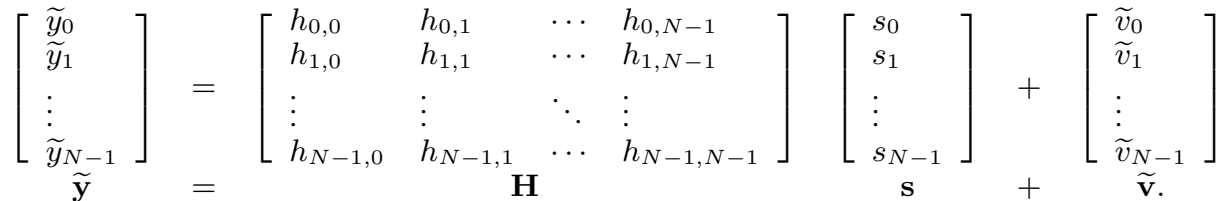

TABLE IV: Pseudocode for the proposed SIC.

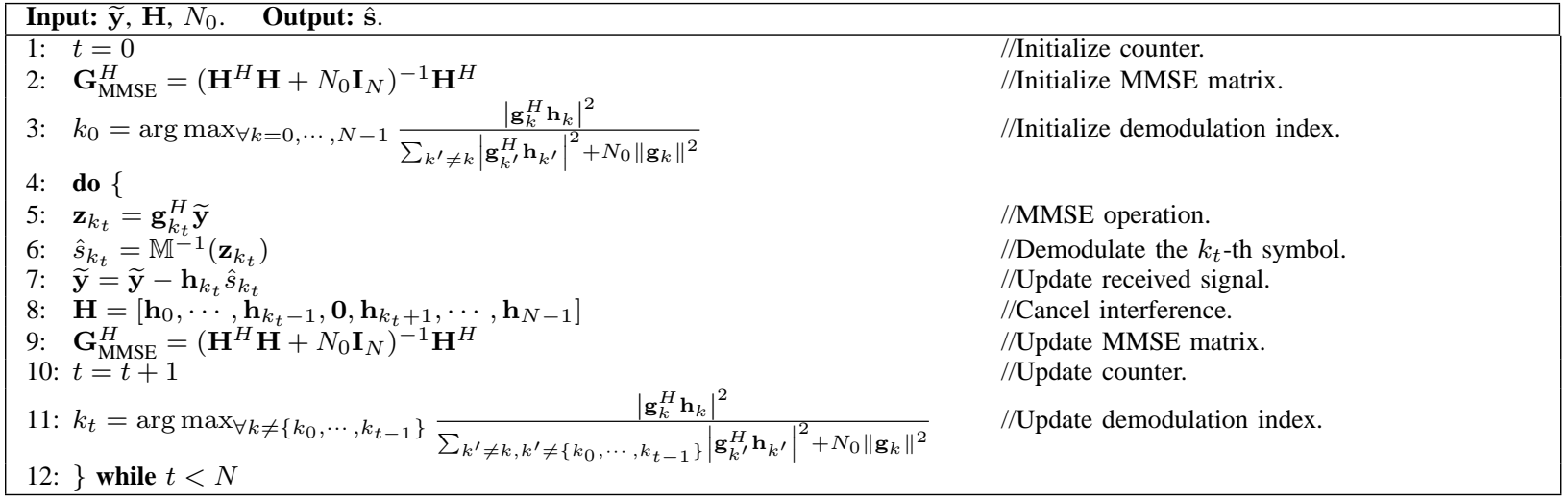

and output distributions. Taking into account the pilot percentage required by FD-CE-TV, the CCMC capacity is given by: $[4],[60],[61]$

$$
C^{\mathrm{CCMC}-\mathrm{FD}}(\mathrm{SNR})=\frac{\zeta^{\mathrm{FD}}}{N} \sum_{k=0}^{N-1} E\left[\log _{2}\left(1+\frac{\left|h_{i, k}\right|^{2}}{N_{0}+N_{0}^{\beta_{i, k}}}\right)\right],
$$

where the FD normalization factor is given by $\left[\zeta^{\mathrm{FD}}=\right.$ $\left.\frac{N_{\mathrm{PS}}^{\text {time }} N-N_{p}^{\text {freq }}}{N_{\mathrm{PS}}^{\text {time }}\left(N+N_{c p}\right)}\right]$, while the ICI power $N_{0}^{\beta_{i, k}}=E\left(\left|\beta_{i, k}\right|^{2}\right)$ is expressed as:

$N_{0}^{\beta_{i, k}}=\Re\left\{\frac{1}{N^{2}} \sum_{k^{\prime} \neq k} \sum_{n_{1}=0}^{N-1} \sum_{n_{2}=0}^{N-1} E\left(h_{i, n_{1}, k^{\prime}} h_{i, n_{2}, k^{\prime}}^{*} \omega_{N}^{\left(n_{1}-n_{2}\right)\left(k^{\prime}-k\right)}\right)\right\}$ $=\Re\left\{\frac{1}{N^{2}} \sum_{k^{\prime} \neq k} \sum_{n_{1}=0}^{N-1} \sum_{n_{2}=0}^{N-1} \sum_{l=0}^{N_{h}-1} E\left(\widetilde{h}_{i, n_{1}, l} \widetilde{h}_{i, n_{2}, l}^{*}\right) \omega_{N}^{\left(n_{1}-n_{2}\right)\left(k^{\prime}-k\right)}\right\}$ $=\Re\left\{\frac{1}{N^{2}} \sum_{k^{\prime} \neq k} \sum_{n_{1}=0}^{N-1} \sum_{n_{2}=0}^{N-1}\left[\widetilde{h}_{i, n_{1}, 0} \widetilde{h}_{i, n_{2}, 0}^{*}\right.\right.$ $\left.\left.+\sum_{l=1}^{N_{h}-1} \widetilde{r}_{l}^{\mathrm{NLoS}}\left(0, n_{1}-n_{2}\right)\right] \omega_{N}^{\left(n_{1}-n_{2}\right)\left(k^{\prime}-k\right)}\right\}$.

In practice, the modulated PSK/QAM symbols constitute a non-Gaussian channel input. In this scenario, the mutual information is maximized, when the modulated PSK/QAM symbols are equiprobable, i.e. we have $\left\{p\left(s^{l}\right)=\frac{1}{L}\right\}_{l=1}^{L}$, where $\left\{s^{l}\right\}_{l=1}^{L}$ refer to the $L$-PSK/QAM constellation points. As a result, the DCMC capacity is given by: [4], [60], [61]

$C^{\mathrm{DCMC}-\mathrm{FP}}(\mathrm{SNR})=\frac{\zeta^{\mathrm{FD}}}{N L} \sum_{k=0}^{N-1} \sum_{\iota=0}^{L-1} E\left\{\log _{2}\left[\frac{L \exp \left(-\frac{\left|y_{i, k}-\hat{h}_{i, k} s^{\iota}\right|^{2}}{N_{0}+N_{0}^{\beta_{i, k}}}\right)}{\sum_{\iota^{\prime}=0}^{L-1} \exp \left(-\frac{\left|y_{i, k}-\hat{h}_{i, k} s^{\iota^{\prime}}\right|^{2}}{N_{0}+N_{0}^{\beta_{i, k}}}\right)}\right]\right\}$ where $\hat{h}_{i, k}$ is obtained by the MMSE solution derived for the FD-CE-TV.

Similarly, based on the TD received signal model of (18), the associated CCMC capacity in the TD is given by:

$$
C^{\mathrm{CCMC}-\mathrm{TD}}(\mathrm{SNR})=\frac{\zeta^{\mathrm{TD}}}{N} \sum_{k=0}^{N-1} E\left[\log _{2}\left(1+\eta_{k}\right)\right],
$$

where the TD normalization factor is formulated as $\left[\zeta^{\mathrm{TD}}=\right.$ $\left.\frac{\left(N_{\mathrm{PS}}^{\text {time }}-1\right) N}{\left(N_{\mathrm{PS}}^{\text {time }}-1\right)\left(N+N_{c p}\right)+2 N_{c p}+1}\right]$, while the SINR $\eta_{k}$ is given by $(45)$. Furthermore, the DCMC capacity of TD-CE-TV is evaluated by:

$C^{\mathrm{DCMC}-\mathrm{TD}}(\mathrm{SNR})=\frac{\zeta^{\mathrm{TD}}}{N L} \sum_{k=0}^{N-1} \sum_{\iota=0}^{L-1} E\left\{\log _{2}\left[\frac{L \exp \left(-\eta_{k}\left|z_{k}-s^{\iota}\right|^{2}\right)}{\sum_{\iota^{\prime}=0}^{L-1} \exp \left(-\eta_{k}\left|z_{k}-s^{\iota^{\prime}}\right|^{2}\right)}\right]\right\}$,

where $z_{k}$ is the $k$-th element in the TD MMSE output vector $\mathbf{z}$ of (43).

\section{Performance Results}

\section{A. Simulation Parameters}

In this section, we examine the performance of the four UAV scenarios portrayed in Fig. 1, where the simulation parameters are summarized in Table V. The parameters choices are elaborated on in terms of the following aspects. 1) In terms of their positioning, the users are assumed to be over $500 \mathrm{~m}$ far away from the BS, where the weak direct BS-user link needs the assistance of RIS. For Scenario D of Fig. 1, the drone is assumed to be flying at a high altitude of $20 \mathrm{~km}$ in the stratosphere, which is above the commercial airplanes [43], [44]. 2) In terms of mobility, the terrestrial BS is always stationary, while both the user and the RIS carried by the UAV may move at a speed that may result in doubly selective Ricean fading, which constitutes one of the major differences between this work and the existing quasi-static solutions. 3) For the Ricean fading, the links emanating from UAVs at an elevated angle are assigned higher $\mathrm{K}$ factors and fewer CIR taps, as seen in Table V. 4) Considering that the smaller OFDM size is often used in high-mobility scenarios, we opt 
TABLE V: Summary of parameters.

\begin{tabular}{|c|c|c|c|c|}
\hline & Scenario A & Scenario B & Scenario C & Scenario D \\
\hline Initial coordinates & $\begin{array}{l}\left(x_{\mathrm{BS}}, y_{\mathrm{BS}}, z_{\mathrm{BS}}\right)=(0,0,0) \\
\left(x_{\mathrm{RIS}}, y_{\mathrm{RIS}}, z_{\mathrm{RIS}}\right)=(500,0,4) \\
\left(x_{\text {User }}, y_{\text {User }}, z_{\text {User }}\right)=(500,-2,0) \\
\end{array}$ & $\begin{array}{l}\left(x_{\mathrm{BS}}, y_{\mathrm{BS}}, z_{\mathrm{BS}}\right)=(0,0,0) \\
\left(x_{\mathrm{RIS}}, y_{\mathrm{RIS}}, z_{\mathrm{RIS}}\right)=(500,0,4), \\
\left(x_{\text {User }}, y_{\text {User }}, z_{\text {User }}\right)=(500,-2,0) \\
\end{array}$ & $\begin{array}{l}\left(x_{\mathrm{BS}}, y_{\mathrm{BS}}, z_{\mathrm{BS}}\right)=(0,0,0), \\
\left(x_{\mathrm{RIS}}, y_{\mathrm{RIS}}, z_{\mathrm{RIS}}\right)=(500,0,10), \\
\left(x_{\text {User }}, y_{\text {User }}, z_{\text {User }}\right)=(500,0,4) \\
\end{array}$ & $\begin{array}{l}\left(x_{\mathrm{BS}}, y_{\mathrm{BS}}, z_{\mathrm{BS}}\right)=(0,0,0), \\
\left(x_{\mathrm{RIS}}, y_{\mathrm{RIS}}, z_{\mathrm{RIS}}\right)=(500,21,20000), \\
\left(x_{\text {User }}, y_{\text {User }}, z_{\text {User }}\right)=(500,20,20000)\end{array}$ \\
\hline Vehicle speeds & $v_{\mathrm{RIS}}=0 \mathrm{mph}, v_{\text {User }}=90 \mathrm{mph}$ & $v_{\mathrm{RIS}}=90 \mathrm{mph}, v_{\text {User }}=90 \mathrm{mph}$ & $v_{\mathrm{RIS}}=0 \mathrm{mph}, v_{\text {User }}=45 \mathrm{mph}$ & $v_{\mathrm{RIS}}=671 \mathrm{mph}, v_{\text {User }}=671 \mathrm{mph}$ \\
\hline Ricean K-factors & $\begin{array}{l}K^{\mathrm{BS}-\text { User }}=3 \mathrm{~dB} \\
K^{\mathrm{BS}-\mathrm{RIS}}=9 \mathrm{~dB} \\
K^{\mathrm{RIS}-\text { User }}=9 \mathrm{~dB}\end{array}$ & $\begin{array}{l}K^{\mathrm{BS}-\text { User }}=3 \mathrm{~dB} \\
K^{\mathrm{BS}-\mathrm{RIS}}=9 \mathrm{~dB} \\
K^{\mathrm{RIS}-\text { User }}=9 \mathrm{~dB}\end{array}$ & $\begin{array}{l}K^{\text {BS-User }}=3 \mathrm{~dB} \\
K^{\mathrm{BS}-\mathrm{RIS}}=9 \mathrm{~dB} \\
K^{\mathrm{RIS}-\text { User }}=12 \mathrm{~dB}\end{array}$ & $\begin{array}{l}K^{\text {BS-User }}=3 \mathrm{~dB} \\
K^{\mathrm{BS}-\mathrm{RIS}}=12 \mathrm{~dB} \\
K^{\mathrm{RIS}-\text { User }}=15 \mathrm{~dB}\end{array}$ \\
\hline CIR taps & 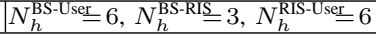 & $N_{h}^{\mathrm{BS}-\mathrm{User}}=6, N_{h}^{\mathrm{BS}-\mathrm{RIIS}}=3, N_{h}^{\mathrm{RIS}-\mathrm{User}}=6$ & $N_{h}^{\mathrm{BS}-\mathrm{User}}=6, N_{h}^{\mathrm{BS}-\mathrm{RIIS}}=3, N_{h}^{\mathrm{RIS}-\mathrm{User}}=3$ & $N_{h}^{\mathrm{BS}-\mathrm{User}}=6, N_{h}^{\mathrm{BS}-\mathrm{RIIS}}=3, N_{h}^{\mathrm{RIS}-\mathrm{User}}=3$ \\
\hline Modulation & \multicolumn{4}{|l|}{$N=64, N_{c p}=8$, QPSK } \\
\hline MMSE parameters & \multicolumn{4}{|c|}{$N_{w}=6, N_{P S}^{f r e q}=8, N_{P S}^{\text {time }}=100 / 50 / 20 / 10 / 2$ for UHF/L/S/C/K/Ka-bands. } \\
\hline Carrier frequency & \multicolumn{4}{|c|}{$0.8 \mathrm{GHz}$ (UHF-band), $1.5 \mathrm{GHz}$ (L-band), $2.6 \mathrm{GHz}$ (S-band), $4.7 \mathrm{GHz}$ (C-band), $26 \mathrm{GHz}$ (K-band), $28.5 \mathrm{GHz}$ (Ka-band) } \\
\hline Bandwidth & \multicolumn{4}{|l|}{$100 \mathrm{kHz}$} \\
\hline Path loss factors & \multicolumn{4}{|c|}{$\gamma^{\mathrm{BS}-\mathrm{User}}=3.8, \gamma^{\mathrm{BS}-\mathrm{RIS}}=\gamma^{\mathrm{RIS-User}}=2.0$} \\
\hline Transmit antenna aperture & \multicolumn{4}{|l|}{$A_{e}^{\mathrm{BS}}=80 \mathrm{~cm}^{2}$} \\
\hline Receive antenna aperture & \multicolumn{4}{|l|}{$A_{e}^{\text {User }}=40 \mathrm{~cm}^{2}$} \\
\hline Receiver noise power density & \multicolumn{4}{|l|}{$-174 \mathrm{dBm} / \mathrm{Hz}$} \\
\hline
\end{tabular}

for choosing $(N=64)$ and $\left(N_{c p}=8\right)$. 5) For the MMSE channel estimation methods, the pilot spacing in the TD is reduced as the carrier frequency increases, due to the increased normalized Doppler, while the pilot spacing in the FD remains the same. 6) The proposed scheme is extensively examined in the 5G NR licensed bands, namely the UHF-band, L-band, Sband, C-band, K-band and Ka-band [39], [40]. 7) The system is assumed to be assigned a $100 \mathrm{kHz}$ bandwidth, which is sufficient for high-mobility Command \& Control $(\mathrm{C} \& \mathrm{C})$ link coverage [44], [45]. 8) Finally, for the PLs of (1), (6) and (11), the PL exponents of the blocked direct link as well as the reflected links are given by $\left(\gamma^{\text {BS-User }}=3.8\right)$ and $\left(\gamma^{\text {BS-RIS }}=\gamma^{\text {RIS-User }}=2.0\right)$, respectively, while the antenna apertures are fixed as $\left(A_{e}^{\mathrm{BS}}=80 \mathrm{~cm}^{2}\right)$ and $\left(A_{e}^{\mathrm{User}}=40 \mathrm{~cm}^{2}\right)$ at the BS and user, respectively. The receiver's noise power density is assumed to be $-174 \mathrm{dBm} / \mathrm{Hz}$. The required transmit power $P_{t}$ in $\mathrm{dBm}$ is evaluated by adding the required $\mathrm{SNR}$ to the noise power of $-174+50=-124 \mathrm{dBm}$. A maximum reference BS transmit power of $P_{t}=30 \mathrm{dBm}$ will be marked in the simulation results. This BS power reference does not apply to the GS for the high-altitude UAVs of Scenario D in Fig. 1, which generally requires a higher power to compensate for the excessive PL.

\section{B. BER Performance Comparison Based on Perfect CSI}

The modelling of RIS assisted OFDM in doubly selective Ricean fading is examined in Fig. 3 for Scenario A of Table V, where the idealistic perfect CSI is temporarily assumed. First of all, Fig. 3 confirms that the employment of RIS is capable of providing substantial performance improvements over the case of having only the direct link, where the RIS gain is increased with the number of RIS elements, as demonstrated by Fig. 3 . Secondly, Fig. 3 also confirms that the RIS gain decreases as the carrier frequency increases, which is due to the increased normalized Doppler and the reduced PL difference between the direct and the reflected links. More explicitly, Fig. 3(a) demonstrates that in the UHF-band, a very substantial $28 \mathrm{~dB}$ performance gain is offered by $(M=4)$ RIS elements at $B E R=10^{-4}$, which is reduced to $25 \mathrm{~dB}$ in the L-band of Fig. 3(b) and to $18 \mathrm{~dB}$ in the S-band of Fig. 3(c). Consequently, we opt for employing the larger $(M=16)$ and $(M=256)$ RIS configurations for the C-band of Fig. 3(d), K-band of Fig. 3(e) and Ka-band of Fig. 3(f), where the performance improvement is still reduced to $21 \mathrm{~dB}, 12 \mathrm{~dB}$ and $5 \mathrm{~dB}$, respectively. Finally, despite relying on the idealistic perfect
CSI, the FD and TD signal detections still suffer from ICI and ISI, where the ICI increases with the Doppler frequency normalized by the carrier frequency. As a result, Figs. 3(e) and 3(f) demonstrate that the FD signal detection experiences irreducible error floors in the K-band and Ka-band, where the TD signal detection offers more reliable performance results.

We note that the thickness of RIS is typically on the order of the subwavelength, which decreases as the operating frequency increases. Therefore, the size of RIS using more elements in higher frequency bands still remains practical. However, at the time of writing, the RIS hardware implementations based on PIN have been mostly tested below or around 10 GHz. Moreover, the micro-electromechanical system (MEMS) implementations are deemed to have an upper limit of 120 GHz. In general, as the carrier frequency is increased, the RIS hardware design and implementation become more challenging, where many parasitic interactions and practical imperfections that have been ignored in the lower frequency bands need to be taken into account [46]-[50]. Therefore, Fig. 3 confirms that the RIS gain decreases as the carrier frequency increases. Hence we may conclude that the RISs are more beneficial in lower frequency bands, especially in high-mobility applications.

\section{MSE and Complexity of FD-CE-TV and TD-CE-TV}

Fig. 4 compares the conventional DFT-based method conceived for time-invariant fading [26] as well as the proposed FD-CE-TV and TD-CE-TV in terms of both their normalized CSI estimation MSE and their complexity. On one hand, Fig. 4(a) demonstrates that the conventional method exhibits the highest level of CSI estimation MSE in the face of doubly selective fading, while the MSE is substantially reduced by both FD-CE-TV and TD-CE-TV upon incrasing $N_{w}$. Explicitly, FD-CE-TV achieves the lowest MSE at each $N_{w}$. On the other hand, Fig. 4(b) also demonstrates that the conventional DFT-based method has the lowest complexity, while the complexities of both the FD-CE-TV and TD-CETV schemes increase with $N_{w}$, where TD-CE-TV exhibits the highest complexity. Based on the MSE and complexity tradeoff portrayed in Fig. 4, we opt to choose $N_{w}=6$ for the proposed FD-CE-TV and TD-CE-TV for the BER and capacity simulation results in the rest of this paper. 


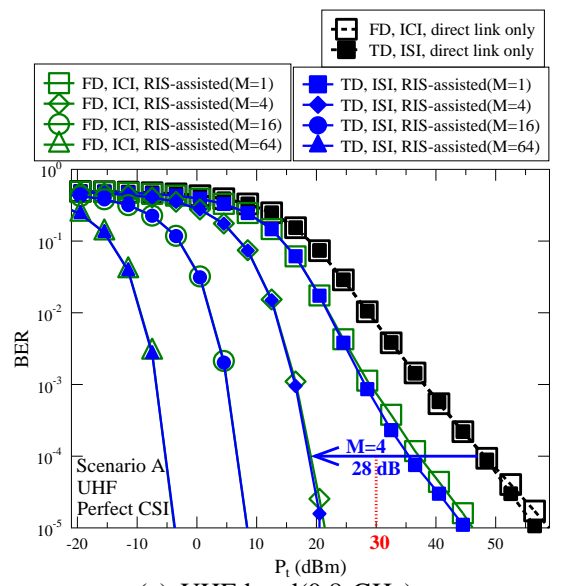

(a) UHF-band $(0.8 \mathrm{GHz})$

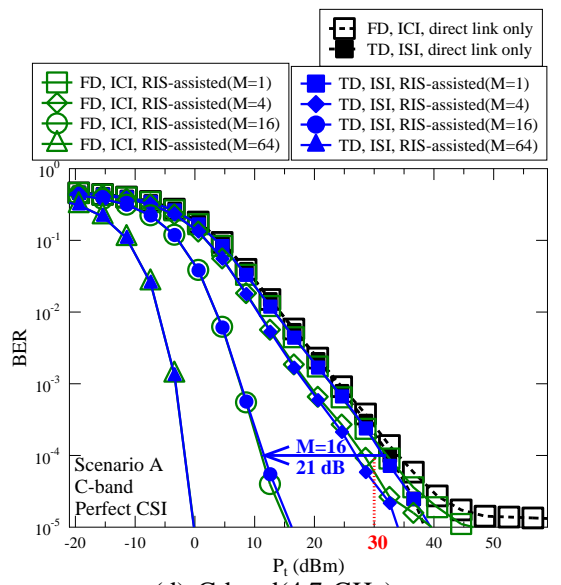

(d) $\mathrm{C}$-band $(4.7 \mathrm{GHz})$

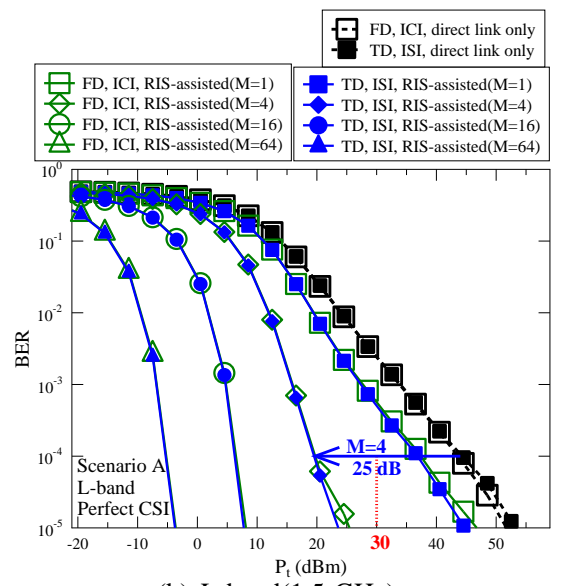

(b) L-band $(1.5 \mathrm{GHz})$

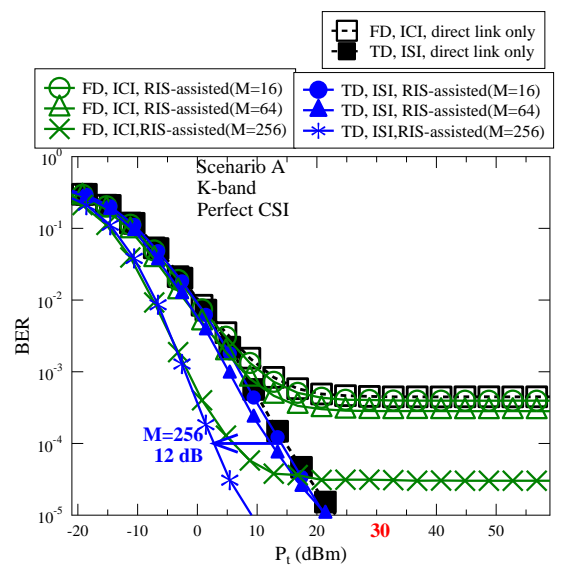

(e) K-band (26 GHz)

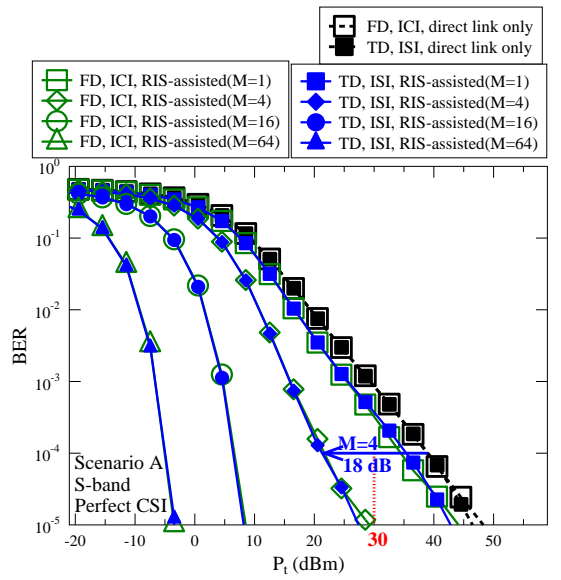

(c) S-band (2.6 GHz)

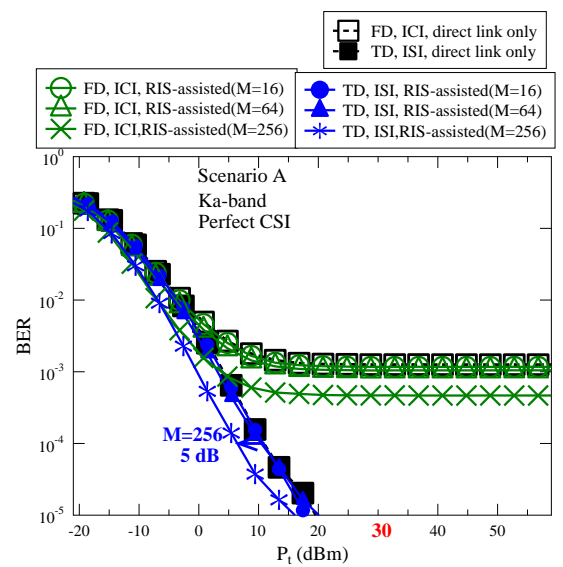

(f) Ka-band(38.5 GHz)

Fig. 3: BER results for RIS assisted OFDM $\left(N=64, N_{c p}=8\right)$ over doubly selective Ricean fading of $\mathrm{Scenario} \mathrm{A}\left(v_{R}=0 \mathrm{mph}, v_{D}=90\right.$ $\mathrm{mph})$, where perfect CSI is assumed. The parameters are specified in Table V.

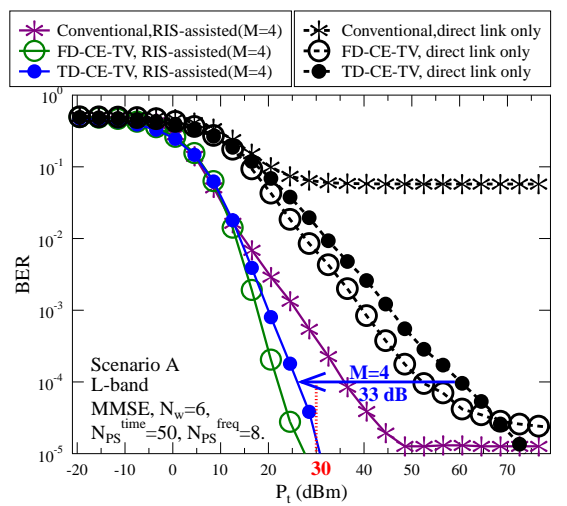

(a) Scenario A, L-band $(1.5 \mathrm{GHz})$

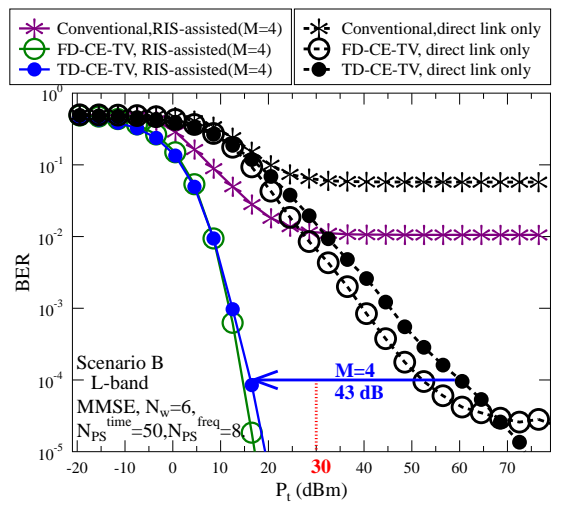

(d) Scenario B, L-band(1.5 GHz)

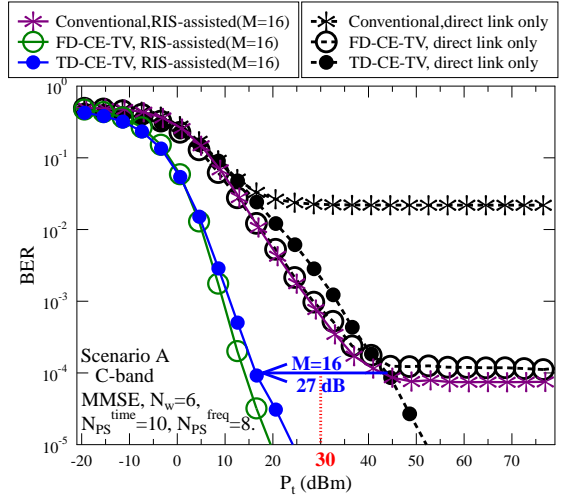

(b) Scenario A, C-band $(4.7 \mathrm{GHz})$

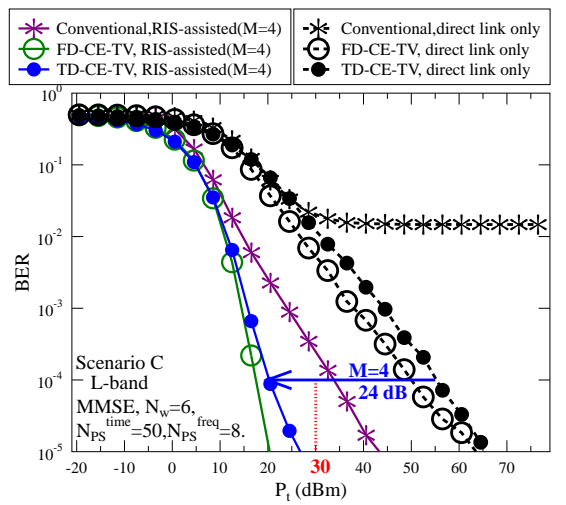

(e) Scenario C, L-band $(1.5 \mathrm{GHz})$

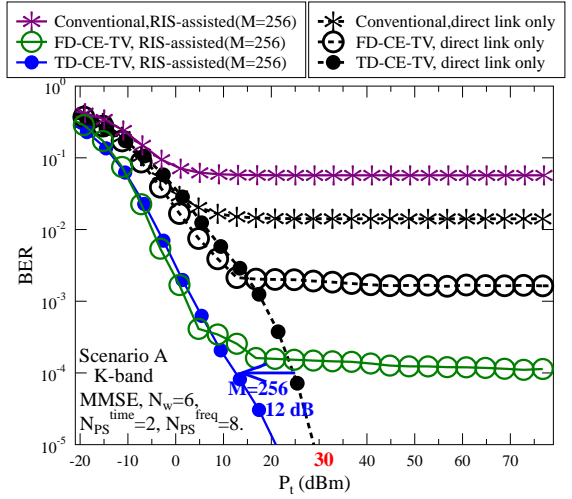

(c) Scenario A, K-band $(26 \mathrm{GHz})$

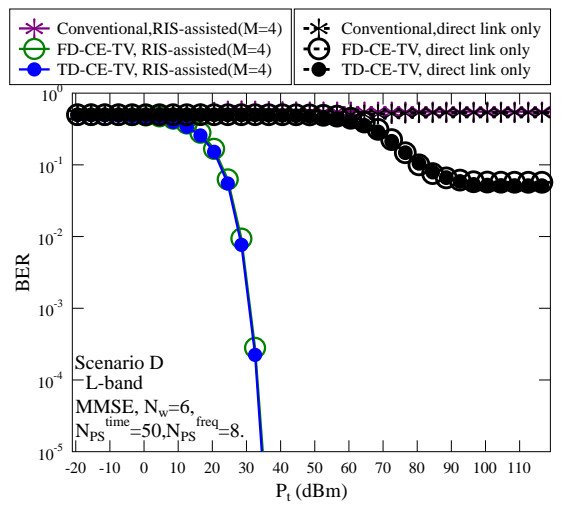

(f) Scenario D, L-band $(1.5 \mathrm{GHz})$

Fig. 5: BER performance results for RIS assisted OFDM over doubly selective Ricean fading, where the proposed FD-CE-TV and TD-CE-TV are invoked. The parameters are specified in Table V. 


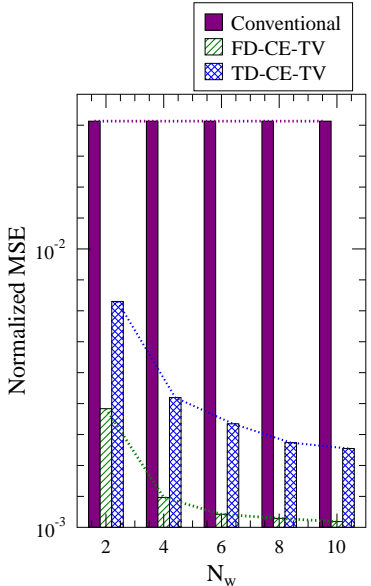

(a) Normalized MSE

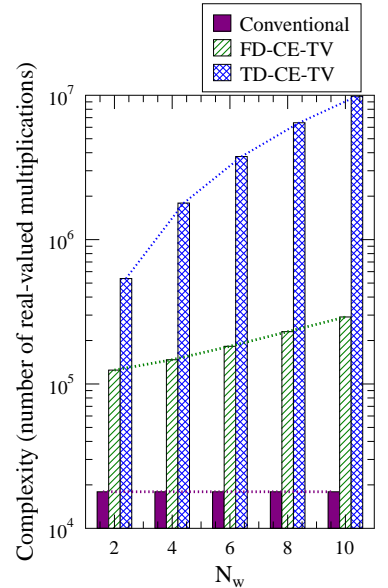

(b) Complexity
Fig. 4: Normalized MSE and complexity comparison between the conventional DFT-based method conceived for time-invariant fading [26] and the proposed FD-CE-TV and TD-CE-TV conceived for timevarying fading. The results are recorded at $P_{t}=30 \mathrm{dBm}$ for Scenario $\mathrm{A}$ in L-band $(1.5 \mathrm{GHz})$. The parameters are specified in Table V.

\section{BER Performance of FD-CE-TV and TD-CE-TV}

Fig. 5 portrays the BER performance results of the proposed FD-CE-TV and TD-CE-TV for RIS assisted OFDM transmissions over doubly selective Ricean fading channels. The conventional DFT-based channel estimation method conceived for time-invariant fading in [26] is used as our benchmark in Fig. 5.

First of all, Fig. 5 demonstrates that the benchmark of the conventional RIS channel estimation technique imposes a performance loss, which results in an irreducible error floor, when the carrier frequency is increased in Figs. 5(b) and 5(c) and the mobility is increased in Figs. 5(d) and 5(f). By contrast, the proposed FD-CE-TV and TD-CE-TV schemes are capable of achieving RIS gains over their respective counterparts of using only the direct link, as confirmed by Figs. 5(a)(f). Secondly, when comparing the results of scenario A in the L-band of Fig. 5(a), C-band of Fig. 5(b) and K-band of Fig. 5(c), it becomes clear that the RIS gain decreases as the carrier frequency is increased, despite using more RIS elements in the higher frequency bands. Thirdly, the RIS gain seen in Scenario B of Fig. 5(d) is $53 \mathrm{~dB}$, which is even more substantial than the $33 \mathrm{~dB}$ achieved in Scenario A of Fig. 5(a). This is due to the fact that the fixed-wing UAV is moving in the same direction at the same speed as the vehicle on the ground in Scenario B, which offers a more reliable RIS-User link. Fourthly, it is once again confirmed by Fig. 5(c) that the Doppler-dependent ICI results in an error floor for FDCE-TV in the high K-band. Finally, Figs 5(a)-(e) demonstrate that when considering $P_{t}=30 \mathrm{dBm}$ as a reference maximum BS transmit power, the employment of RIS is capable of achieving the target of BER $=10^{-4}$ within the BS's coverage, which cannot be achieved by the direct BS-User link operating without RIS. Moreover, Fig. 5(f) demonstrates that when airframe fading is encountered at an aircraft speed of 671 $\mathrm{mph}$, the direct link without RIS exhibits an error floor. This is in line with the findings of [44], [51], confirming that the airframe-induced fading is highly detrimental. Nonetheless,
Fig. 5(f) shows that the FD-CE-TV and TD-CE-TV proposed for RIS assisted OFDM is capable of helping the high-altitude UAV to maintain a reliable link to the GS.

In summary, with the aid of the proposed FD-CE-TV and TD-CE-TV, RIS assisted OFDM systems communicating over doubly selective Ricean fading perform better, especially in the low frequency bands, where maintaining the reliability of the RIS-User link of Scenario B is shown to be more beneficial.

\section{E. CCMC Capacity}

Fig. 6 portrays the CCMC capacity of RIS assisted OFDM over doubly selective Ricean fading channels, where temporarily perfect CSI is assumed. First of all, it is demonstrated by Figs. 6(a)-(c) that even in the L-band, the FD ICI imposes a capacity limit compared to the TD capacity results. This phenomenon is not reflected by the BER results and DCMC capacity results, because QPSK modulation is used, which has the maximum achievable rate of $2 \mathrm{bits} / \mathrm{sec} / \mathrm{Hz}$. Secondly, Figs. 6(a)-(c) confirm that the employment of RIS is capable of achieving a beneficial capacity gain, which improves with the number of RIS elements. Thirdly, upon comparing Scenario A in L-band of Fig. 6(a) and Scenario B in L-band of Fig. 6(b), the latter shows a higher RIS capacity gain, which is a substantial $3.6 \mathrm{bits} / \mathrm{sec} / \mathrm{Hz}$ gain at $P_{t}=30 \mathrm{dBm}$ for $(M=4)$. This confirms our earlier observation that securing the reliability of the RIS-User link of Scenario B is more beneficial. Finally, Fig. 6(c) demonstrates that in the face of the grave airframe fading, the direct link exhibits near-zero capacity owing to severe blockage in the absence of RISs. By contrast, the employment of RISs is capable of mitigating airframe fading, as shown in Fig. 6(c).

In summary, the CCMC capacity results confirm the benefits of RIS deployment based on the BER performance results. Moreover, the FD input-output channel model is shown to have a CCMC capacity limit even in the low frequency bands, which is imposed by the severe ICI of high-mobility applications.

\section{F. DCMC Capacity}

Fig. 7 presents the DCMC capacity of RIS assisted OFDM in doubly selective Ricean fading, where the proposed FDCE-TV and TD-CE-TV are invoked. In contrast to the CCMC capacity that relies on Guassian-distributed input signals, the DCMC capacity relies on the discrete-valued QPSK input constellation, where the achievable rates are normalized by pilot overhead of FD-CE-TV and TD-CE-TV, as formulated in Sec. IV-C. Fig. 7 demonstrates that the proposed FD-CETV and TD-CE-TV perform closely to their counterparts using idealistic perfect CSI. Furthermore, Fig. 7 once again confirms our previous BER and CCMC capacity trends, namely that the employment of RIS is capable of offering substantial performance improvements over the scenario of using only the direct link without RISs.

\section{G. The Effects of RIS Configuration and Channel Estimation}

Fig. 8 demonstrates that in the LoS-dominated UAV scenarios, the performance results of RIS configured based on 


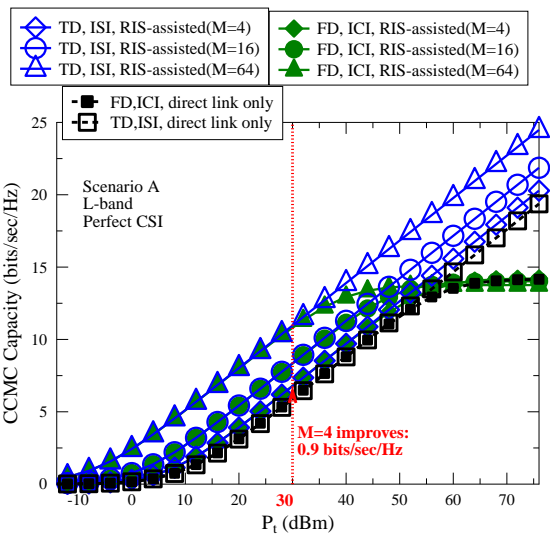

(a) Scenario A, L-band $(1.5 \mathrm{GHz})$

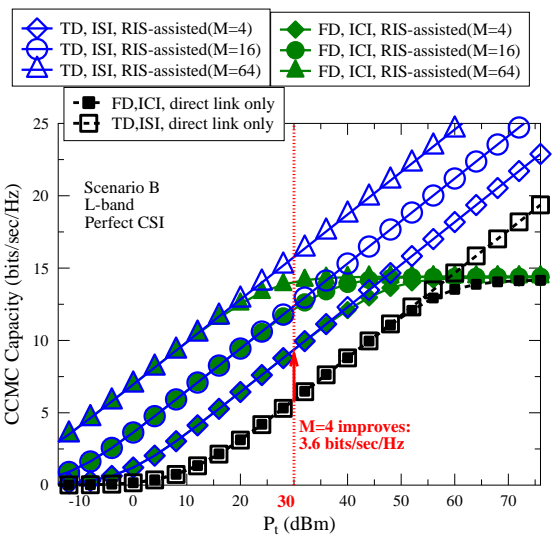

(b) Scenario B, L-band(1.5 GHz)

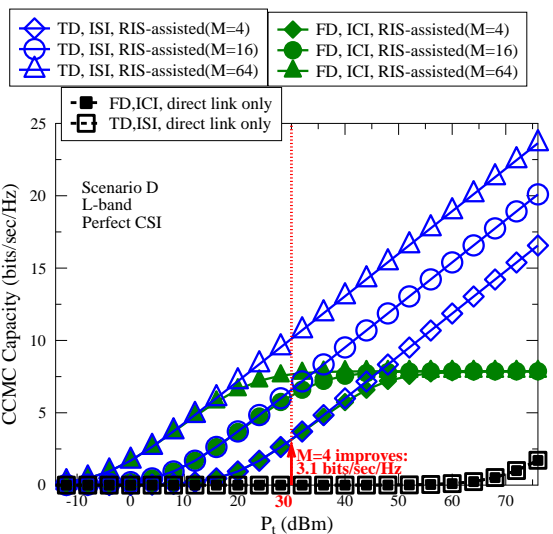

(c) Scenario D, L-band $(1.5 \mathrm{GHz})$

Fig. 6: CCMC capacity results for RIS assisted OFDM over doubly selective Ricean fading, where perfect CSI is assumed. The parameters are specified in Table V.

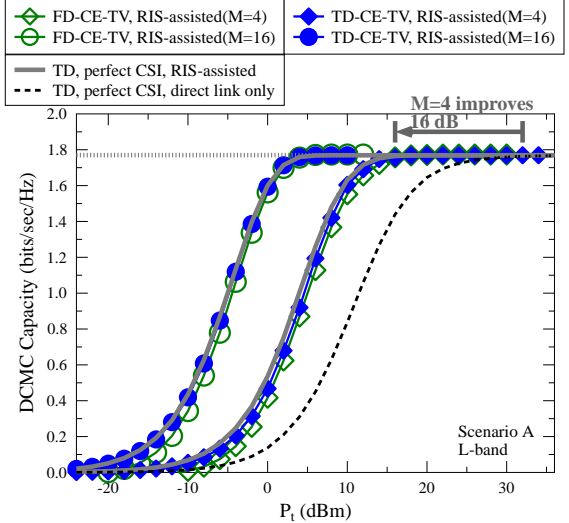

(a) Scenario A, L-band $(1.5 \mathrm{GHz})$

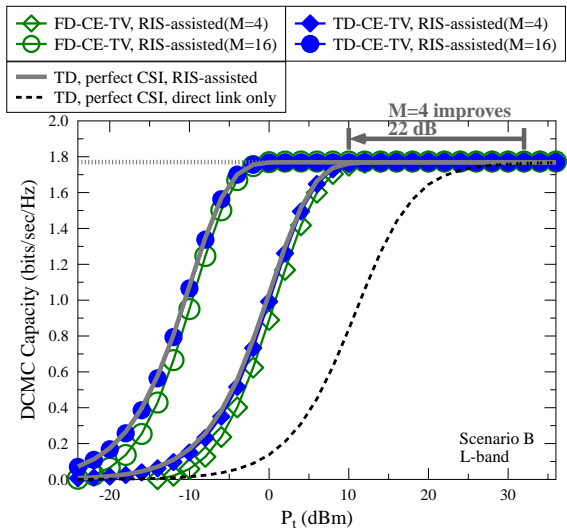

(b) Scenario B, L-band $(1.5 \mathrm{GHz})$

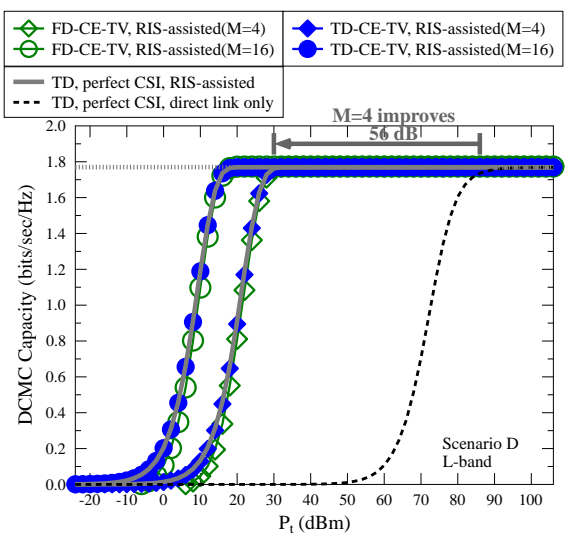

(c) Scenario D, L-band $(1.5 \mathrm{GHz})$

Fig. 7: DCMC capacity results for RIS assisted OFDM over doubly selective Ricean fading, where the proposed FD-CE-TV and TD-CE-TV are invoked. The parameters are specified in Table V.

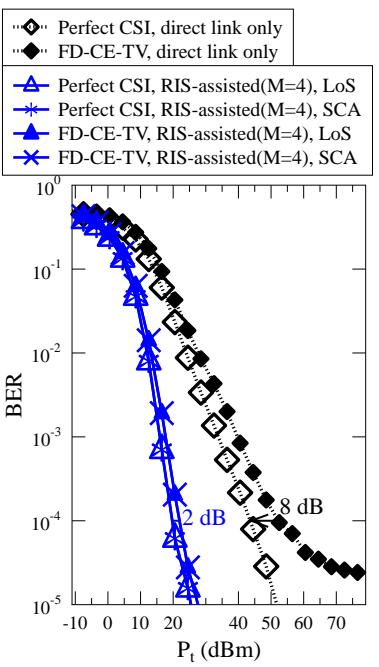

(a) FD-CE-TV

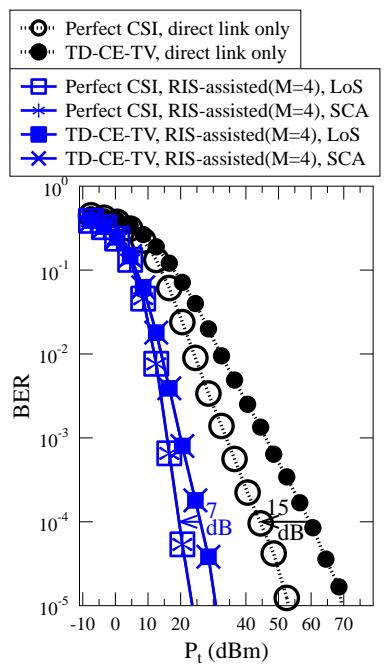

(b) TD-CE-TV

Fig. 8: BER performance results for different RIS configurations for Scenario A in L-band $(1.5 \mathrm{GHz})$, where the proposed FD-CE-TV and TD-CE-TV are compared to their idealistic performance bounds of using perfect CSI.

LoS show negligible difference to the performance results of RIS configured based on the SCA techniques [23]. We note that the motivation of deploying RIS is to create the favourable LoS propagation environment, and optimizing RIS based on LoS is shown to be an effective approach. Furthermore, Figs. 8(a) and (b) demonstrate that the gap between the realistic channel estimation techniques and their idealistic

performance bounds of using perfect CSI is reduced when RIS is deployed. Explicitly, the gap is shown to be $8 \mathrm{~dB}$ and $15 \mathrm{~dB}$ at BER $=10^{-4}$ for FD-CE-TV and TD-CE-TV, respectively, when only direct link is used, but the gap is reduced to $2 \mathrm{~dB}$ and $7 \mathrm{~dB}$ for FD-CE-TV and TD-CE-TV in Fig. 8(a) and Fig. 8(b), respectively, when RIS is deployed. This is due to the fact that channel estimation becomes more accurate at higher SNRs, and the deployment of RIS effectively improves the receive SNR.

\section{H. Effect of Vehicle Speed}

The BER performance of the conventional DFT-based method conceived for time-invariant fading [26] and the proposed TD-CE-TV shown in Fig 5(b) and Fig. 5(d) are extended to 3-D associated with different vehicle speeds in Fig. 9(a) and Fig. 9(b), respectively. It can be seen in Fig. 9 that the conventional DFT-based method conceived for timeinvariant fading [26] begins to experience error floor at 60 mph for both scenarios. Explicitly, the proposed TD-CE-TV is capable of mitigating the effect of doubly selective fading as $v_{D}$ continues to increase to the high-speed train velocity of 180 mph. We note that it was also demonstrated by Fig. 5(f) that the proposed TD-CE-TV is capable of adequately operating even at an airplane speed of $671 \mathrm{mph}$.

\section{Effect of Distance}

Fig. 10 investigates the power-efficiency gain of using RISs, when the user is travelling to different locations for 


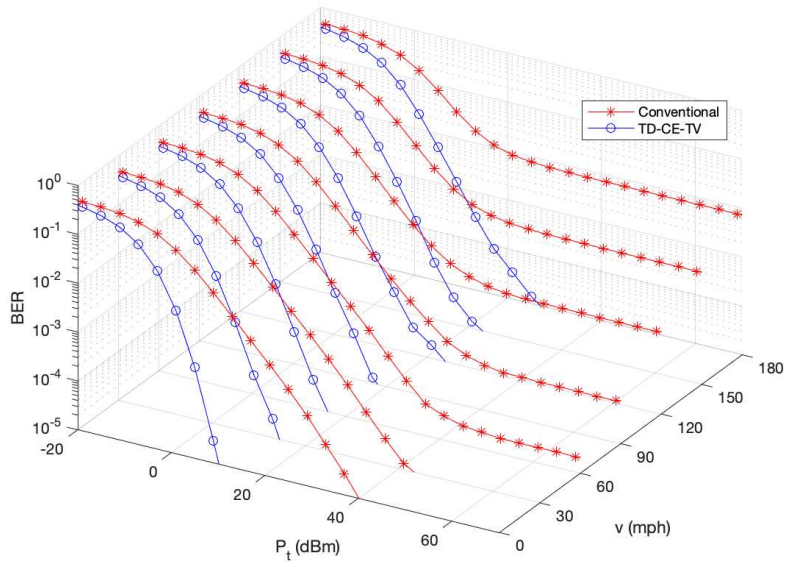

(a) Scenario A, C-band $(4.7 \mathrm{GHz})$

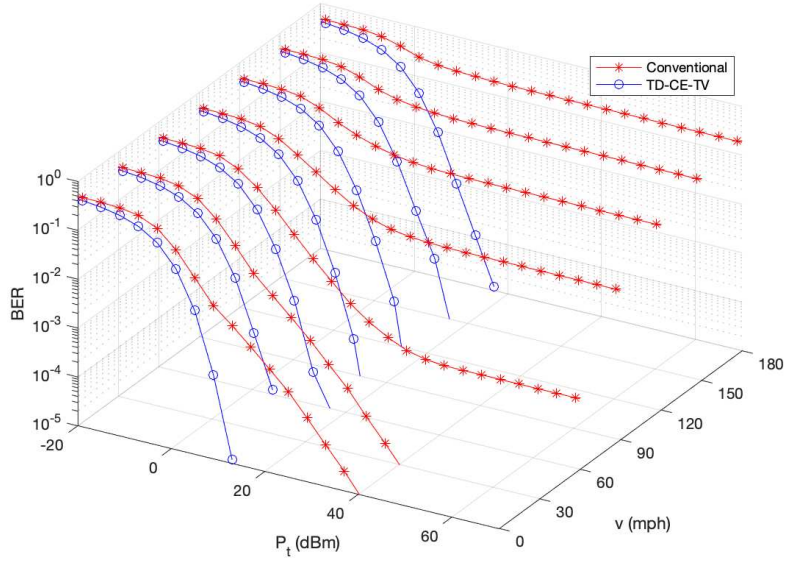

(b) Scenario B, L-band $(1.5 \mathrm{GHz})$

Fig. 9: 3-D BER performance results with respect to SNR and vehicle speed for RIS assisted OFDM over doubly selective Ricean fading, where the conventional DFT-based method conceived for time-invariant fading [26] and the proposed TD-CE-TV are invoked.

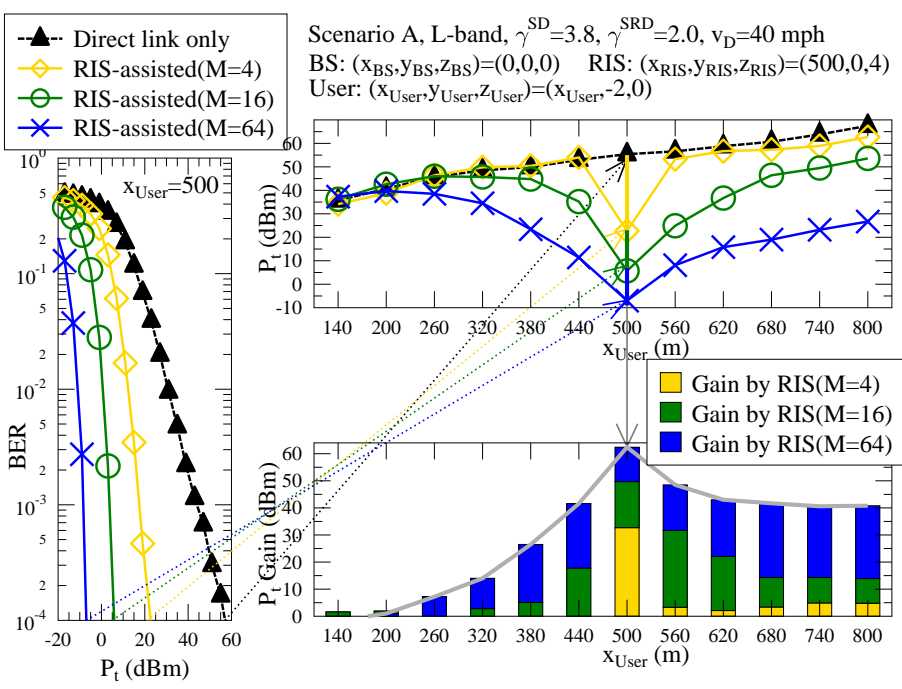

Fig. 10: The power-efficiency gain of using RIS in terms of the required transmit power $P_{t}(\mathrm{dBm})$ for achieving target BER of $10^{-4}$, when the user is travelling to different locations of $\left(x_{D}, y_{D}, z_{D}\right)=$ $\left(x_{D},-2,0\right)$ for Scenario A in L-band $(1.5 \mathrm{GHz})$. The proposed TDCE-TV is invoked.

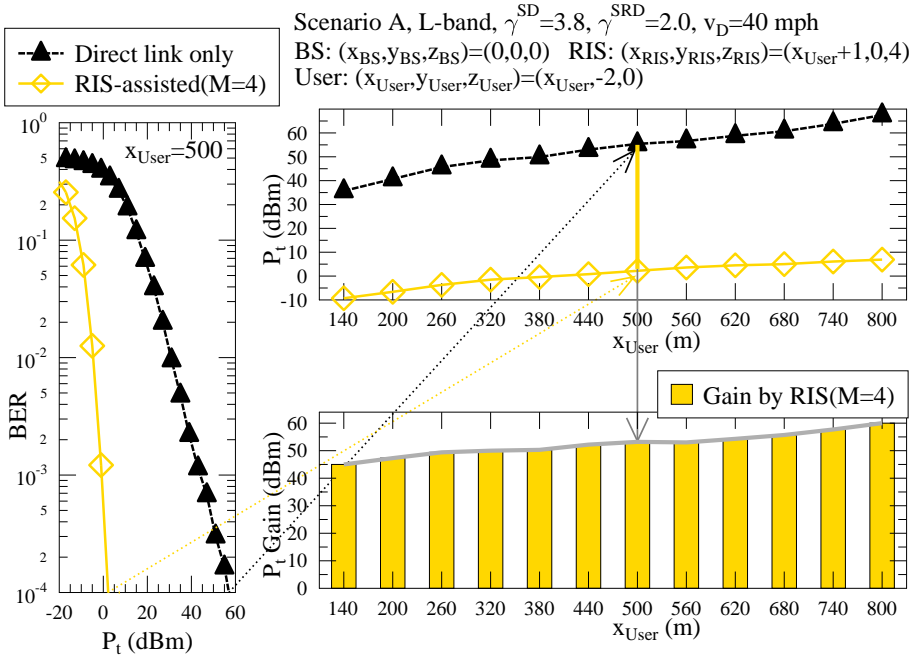

Fig. 11: The power-efficiency gain of using RIS in terms of the required transmit power $P_{t}(\mathrm{dBm})$ for achieving target BER of $10^{-4}$, when the user is travelling to different locations of $\left(x_{D}, y_{D}, z_{D}\right)=$ $\left(x_{D},-2,0\right)$ with a transparent RIS on car window $\left(x_{R}, y_{R}, z_{R}\right)=$ $\left(x_{D}-1,-2,0\right)$ for Scenario A in L-band $(1.5 \mathrm{GHz})$. The proposed TD-CE-TV is invoked.

Scenario A and communicating in the L-band (1.5 GHz). More explicitly, the transmit powers $P_{t}(\mathrm{dBm})$ for $\mathrm{BER}=10^{-4}$ at different user locations of $\left(x_{D}, y_{D}, z_{D}\right)=\left(x_{D},-2,0\right)$ are recorded at the top right subfigure of Fig. 10, while the powerefficiency gains are recorded in the bottom right subfigure. Fig. 10 demonstrates that the RIS achieves a higher powerefficiency gain, when the user is closer to the RIS location of $\left(x_{R}, y_{R}, z_{R}\right)=(500,0,4)$. On one hand, when the user moves closer to the BS's location of $\left(x_{S}, y_{S}, z_{S}\right)=(0,0,0)$, the direct SD link becomes strong enough so that harnessing the RIS gradually loses its advantage. On the other hand, when the user moves away from both the BS and the RIS, the transmit power required by the RIS assisted scheme also increases, since both the direct link and the RIS-reflected link become weaker. Nonetheless, Fig. 10 confirms that by using a RIS associated with the increased size of $M=64$, the proposed TD-CE-TV aided RIS system becomes capable of reducing the required $P_{t}$ below the maximum of $30 \mathrm{dBm}$ for a wider range of user locations all the way up to $\left(x_{D}=800\right) \mathrm{m}$.

\section{J. Coverage Extension by Transparent RIS}

The coverage of Fig. 10 may be further improved by the recent development of transparent metasurfaces [62], [63], which can achieve both $0^{\circ} \sim 180^{\circ}$ reflection and $180^{\circ} \sim 360^{\circ}$ refraction. Therefore, the transparent RIS may be superimposed on a car window, so that the RIS and the receive antenna inside the car are close to each other and also stationary with respect to each other. In order to reflect on this recent development, the coverage extension attained by the transparent RISs is investigated in Fig. 11, where we have $f_{D}^{\text {BS-User }}=f_{D}^{\text {BS-RIS }}=\frac{v f_{c}}{c}$ and $f_{D}^{\text {RIS-User }}=0$. The RIS is assumed to be within 1 meter from the receive antenna. As a result, Fig. 11 demonstrates that a small RIS using as few as $M=4$ elements is sufficient for reducing the required $P_{t}$ well below the maximum limit of $P_{t}=30 \mathrm{dBm}$ across the 
wide range of user locations spanning from $\left(x_{D}=140\right) \mathrm{m}$ to $\left(x_{D}=800\right) \mathrm{m}$.

\section{K. Further Discussions}

As an alternative to OFDM, the recently developed orthogonal time frequency space (OTFS) modulation [64] is capable of tackling the doubly selective fading by converting the timevarying channels represented in the time-frequency domain by transforming them into the time-invariant channels of the delay-Doppler domain. The OTFS modulates its symbols in the delay-Doppler domain, which is equivalent to a precoded OFDM scheme using the symplectic finite Fourier transform (SFFT) [65]. Therefore, the proposed TD-CE-TV method that performs channel estimation by transmitting Dirac delta signals in the TD can be directly applied, when OTFS is invoked for doubly selective RIS channels. Furthermore, it may be envisioned that channel estimation method tailored for OTFS by sending Dirac delta signals in the delay-Doppler domain [66] may impse a reduced complexity, because observing fading correlation in the TD is no longer neccessary for the timeinvariant fading of the delay-Doppler domain. However, the combination of OTFS and RIS in high-mobility scenarios still faces major challenges. Explicitly, OTFS requires sufficiently long time duration and high bandwidth for accurately locating the signals in the delay-Doppler domain, where the Doppler frequencies are the exact integer multiplies of Doppler taps. However, the control links required for reliable coverage by UAVs [44], airplanes [43] and high-speed trains [67] often operate at low rates in the highly-congested lower frequency bands such as the popular L-band. Furthermore, the signal detection of OTFS may become more complex than that of OFDM, since OFDM's SC orthogonality is no longer exploited. Therefore, RIS-aided OTFS is beyond the scope of this treatise, but it is set aside for our future research.

\section{CONCLUSIONS}

A sophisticated RIS-aided OFDM transmission was conceived for high-mobility scenario in the face of doubly selectivity Ricean fading, where the RIS control feedback link exploited a priori knowledge about LoS statistics. Our new MMSE channel estimation methods of FD-CE-TV and TDCE-TV operating in the FD and TD, respectively, supported high-integrity for our high-dimensional RIS-aided OFDM system. A low-complexity RIS configuration algorithm was proposed for maximizing the received signal power at the high-mobility user. Moreover, new interference mitigation techniques were conceived for improving the performance.

Our simulation results confirm the following observations. Firstly, the employment of RIS is capable of achieving substantial performance improvements over the scenario of using only the direct link without RISs. Secondly, the RIS gain decreases as the carrier frequency increases, which is due to the increased normalized Doppler frequency and the reduced path loss difference between the direct and the reflected links. Thirdly, improving the RIS-User link of the reflected BS-RISUser link is shown to be more beneficial than that of the BS-RIS link. Finally, at high carrier frequencies, TD signal processing is preferred, because the ICI encountered by FD signal detection increases with the carrier frequency.

\section{REFERENCES}

[1] R. Steele and L. Hanzo, Mobile Radio Communications. John Wiley \& Sons, May 1999.

[2] J. G. Proakis, Digital Communications. New York: McGraw-Hill, 1995.

[3] A. Goldsmith, Wireless communications. Cambridge University Press, 2005.

[4] L. Hanzo, O. Alamri, M. El-Hajjar, and N. Wu, Near-Capacity MultiFunctional MIMO Systems: Sphere-Packing, Iterative Detection and Cooperation. John Wiley \& Sons, May 2009.

[5] L. Hanzo, S. X. Ng, T. Keller, and W. Webb, Quadrature Amplitude Modulation: From Basics to Adaptive Trellis-Coded, Turbo-Equalised and Space-Time Coded OFDM, CDMA and MC-CDMA Systems. John Wiley \& Sons, 2004.

[6] L. Hanzo, T. Liew, B. Yeap, R. Tee, and S. X. Ng, Turbo Coding, Turbo Equalisation and Space-Time Coding: EXIT-Chart-Aided Near-Capacity Designs for Wireless Channels. John Wiley \& Sons, 2011.

[7] L. Hanzo, Y. Akhtman, L. Wang, and M. Jiang, MIMO-OFDM for LTE, WiFi and WiMAX: Coherent versus Non-coherent and Cooperative Turbo Transceivers. Wiley-IEEE Press, 2011.

[8] M. D. Renzo, M. Debbah, D. T. Phan-Huy, A. Zappone, M. S Alouini, C. Yuen, V. Sciancalepore, G. C. Alexandropoulos, J. Hoydis, H. Gacanin, and J. D. Rosny, "Smart radio environments empowered by reconfigurable AI meta-surfaces: An idea whose time has come," EURASIP J. Wireless Commun. Netw., 2019.

[9] Q. Wu and R. Zhang, "Towards smart and reconfigurable environment: Intelligent reflecting surface aided wireless network," IEEE Commun. Mag., vol. 58, no. 1, pp. 106-112, 2020.

[10] C. Huang, S. Hu, G. C. Alexandropoulos, A. Zappone, C. Yuen, R. Zhang, M. D. Renzo, and M. Debbah, "Holographic MIMO surfaces for 6G wireless networks: Opportunities, challenges, and trends," IEEE Wireless Commun., vol. 27, no. 5, pp. 118-125, 2020.

[11] N. Shlezinger, G. C. Alexandropoulos, M. F. Imani, Y. C. Eldar, and D. R. Smith, "Dynamic metasurface antennas for $6 \mathrm{G}$ extreme massive MIMO communications," IEEE Wireless Commun., vol. 28, no. 2, pp. 106-113, 2021.

[12] L. Zhang, X. Q. Chen, S. Liu, Q. Zhang, J. Zhao, J. Y. Dai, G. D. Bai, X. Wan, Q. Cheng, G. Castaldi, V. Galdi, and T. J. Cui, "Space-timecoding digital metasurfaces," Nat. Commun., vol. 4334, no. 9, 2018.

[13] H. Yang, X. Cao, F. Yang, J. Gao, S. Xu, M. Li, X. Chen, Y. Zhao, Y. Zeng, and S. Li, "A programmable metasurface with dynamic polarization, scattering and focusing control," Scientific Reports, vol. 6, no. 35692, 2016.

[14] G. C. Alexandropoulos, G. Lerosey, M. Debbah, and M. Fink, "Reconfigurable intelligent surfaces and metamaterials: The potential of wave propagation control for $6 \mathrm{G}$ wireless communications," arXiv preprint arXiv:2006.11136.

[15] G. C. Alexandropoulos, N. Shlezinger, and P. Hougne, "Reconfigurable intelligent surfaces for richscattering wireless communications: Recentexperiments, challenges, and opportunities," arXiv preprint arXiv:2103.04711.

[16] Y. Han, W. Tang, S. Jin, C. Wen, and X. Ma, "Large intelligent surfaceassisted wireless communication exploiting statistical CSI," IEEE Trans. Veh. Technol., vol. 68, no. 8, pp. 8238-8242, 2019.

[17] K. Ying, Z. Gao, S. Lyu, Y. Wu, H. Wang, and M. Alouini, “GMD-based hybrid beamforming for large reconfigurable intelligent surface assisted millimeter-wave massive MIMO," IEEE Access, vol. 8, pp. 1953019539, 2020.

[18] B. Di, H. Zhang, L. Li, L. Song, Y. Li, and Z. Han, "Practical hybrid beamforming with finite-resolution phase shifters for reconfigurable intelligent surface based multi-user communications," IEEE Trans. Veh. Technol., vol. 69, no. 4, pp. 4565-4570, 2020.

[19] J. He, H. Wymeersch, T. Sanguanpuak, O. Silven, and M. Juntti, "Adaptive beamforming design for mmWave RIS-aided joint localization and communication," in 2020 IEEE Wireless Commun. Networking Conf. Workshops (WCNCW), pp. 1-6, 2020.

[20] A. Taha, M. Alrabeiah, and A. Alkhateeb, "Enabling large intelligent surfaces with compressive sensing and deep learning," arXiv preprint arXiv:1904.10136.

[21] S. Liu, Z. Gao, J. Zhang, M. D. Renzo, and M.-S. Alouini, "Deep denoising neural network assisted compressive channel estimation for mmWave intelligent reflecting surfaces," arXiv preprint arXiv:2006.02201. 
[22] G. C. Alexandropoulos and E. Vlachos, "A hardware architecture for reconfigurable intelligent surfaces with minimal active elements for explicit channel estimation," in IEEE Int. Conf. Acoustics, Speech Signal Process. (ICASSP), pp. 9175-9179, 2020.

[23] Y. Yang, B. Zheng, S. Zhang, and R. Zhang, "Intelligent reflecting surface meets OFDM: Protocol design and rate maximization," IEEE Trans. Commun., vol. 68, no. 7, pp. 4522-4535, 2020.

[24] Z. Wang, L. Liu, and S. Cui, "Channel estimation for intelligent reflecting surface assisted multiuser communications: Framework, algorithms, and analysis," IEEE Trans. Wireless Commun., pp. 1-1, 2020.

[25] T. L. Jensen and E. De Carvalho, "An optimal channel estimation scheme for intelligent reflecting surfaces based on a minimum variance unbiased estimator," in IEEE Int. Conf. Acoustics, Speech Signal Process. (ICASSP), pp. 5000-5004, 2020.

[26] B. Zheng and R. Zhang, "Intelligent reflecting surface-enhanced OFDM: Channel estimation and reflection optimization," IEEE Wireless Commun. Lett., vol. 9, no. 4, pp. 518-522, 2020.

[27] Q. Nadeem, H. Alwazani, A. Kammoun, A. Chaaban, M. Debbah, and M. Alouini, "Intelligent reflecting surface-assisted multi-user MISO communication: Channel estimation and beamforming design," IEEE Open J. the Commun.s Society, vol. 1, pp. 661-680, 2020.

[28] C. You, B. Zheng, and R. Zhang, "Channel estimation and passive beamforming for intelligent reflecting surface: Discrete phase shift and progressive refinement," IEEE J. Sel. Areas Commun., 2020.

[29] C. You, B. Zheng, and R. Zhang, "Progressive channel estimation and passive beamforming for intelligent reflecting surface with discrete phase shifts," arXiv preprint arXiv:1912.10646.

[30] S. Lin, B. Zheng, G. C. Alexandropoulos, M. Wen, M. D. Renzo, and F. Chen, "Reconfigurable intelligent surfaces with reflection pattern modulation: Beamforming design and performance analysis," IEEE Trans. Wireless Commun., vol. 20, no. 2, pp. 741-754, 2021.

[31] P. Wang, J. Fang, H. Duan, and H. Li, "Compressed channel estimation for intelligent reflecting surface-assisted millimeter wave systems," IEEE Signal Process. Lett., vol. 27, pp. 905-909, 2020.

[32] J. Chen, Y.-C. Liang, H. V. Cheng, and W. Yu, "Channel estimation for reconfigurable intelligent surface aided multi-user MIMO systems,', arXiv preprint arXiv:1912.03619.

[33] X. Ma, Z. Chen, W. Chen, Z. Li, Y. Chi, C. Han, and S. Li, "Joint channel estimation and data rate maximization for intelligent reflecting surface assisted terahertz MIMO communication systems," IEEE Access, pp. 99565-99581, 2020.

[34] Z. He and X. Yuan, "Cascaded channel estimation for large intelligent metasurface assisted massive mimo," IEEE Wireless Commun. Lett., vol. 9, no. 2, pp. 210-214, 2020

[35] H. Liu, X. Yuan, and Y. A. Zhang, "Matrix-calibration-based cascaded channel estimation for reconfigurable intelligent surface assisted multiuser MIMO," IEEE J. Sel. Areas Commun., pp. 1-1, 2020.

[36] L. Wei, C. Huang, G. C. Alexandropoulos, and C. Yuen, "Parallel factor decomposition channel estimation in RIS-assisted multi-user MISO communication," in 2020 IEEE 11th Sensor Array and Multichannel Signal Processing Workshop (SAM), pp. 1-5, 2020.

[37] L. Wei, C. Huang, G. C. Alexandropoulos, C. Yuen, Z. Zhang, and M. Debbah, "Channel estimation for RIS-empowered multi-user MISO wireless communications," IEEE Trans. Commun., vol. 69, no. 6 pp. 4144-4157, 2021.

[38] G. C. A. K. Chen-Hu and A. G. Armada, "Non-coherent modulation for ris-empoweredmulti-antenna ofdm communications," arXiv preprint arXiv:2103.05547.

[39] 3GPP Technical Report 21.915, "Summary of Rel-15 work items," [Online]. Available: http://www.3 gpp.org/release-15.

[40] C. Xu, N. Ishikawa, R. Rajashekar, S. Sugiura, R. G. Maunder, Z. Wang, L. Yang, and L. Hanzo, "Sixty years of coherent versus non-coherent tradeoffs and the road from $5 \mathrm{G}$ to wireless futures," IEEE Access, vol. 7, pp. 178246-178299, 2019.

[41] EUROCONTROL/FAA Future Communications Study Operational Concepts and Requirements Team, "Communications operating concept and requirements (COCR) for the future radio system," Tech. Rep. 1.0.

[42] C. Xu, J. Zhang, T. Bai, P. Botsinis, R. G. Maunder, R. Zhang, and L. Hanzo, "Adaptive coherent/non-coherent single/multiple-antenna aided channel coded ground-to-air aeronautical communication," IEEE Trans. Commun., vol. 67, no. 2, pp. 1099-1116, 2019.

[43] J. Zhang, T. Chen, S. Zhong, J. Wang, W. Zhang, X. Zuo, R. G. Maunder, and L. Hanzo, "Aeronautical Ad Hoc networking for the internet-abovethe-clouds," Proceedings of the IEEE, vol. 107, no. 5, pp. 868-911, 2019.

[44] C. Xu, T. Bai, J. Zhang, R. Rajashekar, R. G. Maunder, Z. Wang, and L. Hanzo, "Adaptive coherent/non-coherent spatial modulation aided unmanned aircraft systems," IEEE Wireless Commun., vol. 26, no. 4, pp. 170-177, 2019

[45] 3GPP Technical Report 36.777, “Technical specification group radio access network; study on enhanced LTE support for aerial vehicles (release 15)," [Online]. Available: http://www.3gpp.org/ftp//Specs/archive/36_series/36.777/, Jan. 2018

[46] E. Basar, M. Di Renzo, J. De Rosny, M. Debbah, M. Alouini, and R. Zhang, "Wireless communications through reconfigurable intelligent surfaces," IEEE Access, vol. 7, pp. 116753-116773, 2019.

[47] Q. Wu and R. Zhang, "Intelligent reflecting surface enhanced wireless network via joint active and passive beamforming," IEEE Trans. Wireless Commun., vol. 18, no. 11, pp. 5394-5409, 2019.

[48] S. Gong, X. Lu, D. T. Hoang, D. Niyato, L. Shu, D. I. Kim, and Y. Liang, "Towards smart wireless communications via intelligent reflecting surfaces: A contemporary survey," IEEE Commun. Surveys Tut., vol. 22, no. 4, pp. 2283-2314, 2020.

[49] J. Zhang, E. Bjrnson, M. Matthaiou, D. W. K. Ng, H. Yang, and D. J. Love, "Prospective multiple antenna technologies for beyond 5G," IEEE J. Sel. Areas Commun., vol. 38, no. 8, pp. 1637-1660, 2020.

[50] Q. Wu, S. Zhang, B. Zheng, C. You, and R. Zhang, "Intelligent reflecting surface aided wireless communications: A tutorial," arXiv preprint arXiv:2007.02759.

[51] R. Sun, D. W. Matolak, and W. Rayess, "Air-ground channel characterization for unmanned aircraft systems - Part IV: Airframe shadowing,' IEEE Trans. Veh. Technol., vol. 66, pp. 7643-7652, Sept 2017.

[52] I. A. Hemadeh, K. Satyanarayana, M. El-Hajjar, and L. Hanzo, "Millimeter-wave communications: Physical channel models, design considerations, antenna constructions, and link-budget," IEEE Commun. Surveys Tut., vol. 20, pp. 870-913, Secondquarter 2018.

[53] T. S. Rappaport, Y. Xing, O. Kanhere, S. Ju, A. Madanayake, S. Mandal, A. Alkhateeb, and G. C. Trichopoulos, "Wireless communications and applications above $100 \mathrm{GHz}$ : Opportunities and challenges for $6 \mathrm{G}$ and beyond," IEEE Access, 2019.

[54] R. H. Clarke, "A statistical theory of mobile radio reception," Bell Labs Technical Journal, vol. 47, pp. 957-1000, 1968.

[55] W. C. Jakes, Microwave mobile communications. John Wiley \& Sons, 1975.

[56] Ye Li and L. J. Cimini, "Bounds on the interchannel interference of OFDM in time-varying impairments," IEEE Trans. Commun., vol. 49, no. 3, pp. 401-404, 2001

[57] T. Hwang, C. Yang, G. Wu, S. Li, and G. Ye Li, "OFDM and its wireless applications: A survey," IEEE Trans. Veh. Technol., vol. 58, no. 4, pp. 1673-1694, 2009.

[58] Yang-Seok Choi, P. J. Voltz, and F. A. Cassara, "On channel estimation and detection for multicarrier signals in fast and selective rayleigh fading channels," IEEE Trans. Commun., vol. 49, no. 8, pp. 1375-1387, 2001.

[59] C. Huang, R. Mo, and C. Yuen, "Reconfigurable intelligent surface assisted multiuser MISO systems exploiting deep reinforcement learning," IEEE J. Sel. Areas Commun., vol. 38, no. 8, pp. 1839-1850, 2020.

[60] C. Xu, S. Sugiura, S. X. Ng, P. Zhang, L. Wang, and L. Hanzo, "Two decades of MIMO design tradeoffs and reduced-complexity MIMO detection in near-capacity systems," IEEE Access, vol. 5, pp. 18564 $18632,2017$.

[61] S. X. Ng and L. Hanzo, "On the MIMO channel capacity of multidimensional signal sets," IEEE Trans. Veh. Technol., vol. 55, no. 2, pp. 528536,2006

[62] DOCOMO, "Docomo conducts world's first successful trial of transparent dynamic metasurface," [Online]. Available: https://www.nttdocomo.co.jp/english/info/media_center/pr/2020/0117_00.html.

[63] X. Wang, J. Ding, B. Zhang, S. An, G. Zhai, and H. Zhang, "Simultaneous realization of anomalous reflection and transmission at two frequencies using bi-functional metasurfaces," Scientific Reports, vol. 8 , no. 1 , pp. 1-8, 2018

[64] R. Hadani, S. Rakib, M. Tsatsanis, A. Monk, A. J. Goldsmith, A. F Molisch, and R. Calderbank, "Orthogonal time frequency space modulation," in 2017 IEEE Wireless Communications and Networking Conference (WCNC), pp. 1-6, 2017.

[65] W. Shen, D. Dai, J. An, P. Fan, and R. W. Heath, "Channel estimation for orthogonal time frequency space (OTFS) massive MIMO," IEEE Trans. Signal Process., vol. 67, no. 16, pp. 4204-4217, 2019.

[66] P. Raviteja, K. T. Phan, and Y. Hong, "Embedded pilot-aided channel estimation for OTFS in delaydoppler channels," IEEE Trans. Veh. Technol., vol. 68, no. 5, pp. 4906-4917, 2019.

[67] J. Wu and P. Fan, "A survey on high mobility wireless communications: Challenges, opportunities and solutions," IEEE Access, vol. 4, pp. 450476, 2016. 